\title{
Fault-Tolerant Control of a Nonlinear System Actuator Fault Based on Sliding Mode Control
}

\author{
Jing He, ${ }^{1}$ Lin Mi, ${ }^{1}$ Songan Mao, ${ }^{2}$ Changfan Zhang, ${ }^{1}$ and Houguang Chu' \\ ${ }^{1}$ College of Electrical and Information Engineering, Hunan University of Technology, Zhuzhou, Hunan 412007, China \\ ${ }^{2}$ School of Electrical and Computer Engineering, Purdue University, West Lafayette, IN 47907, USA \\ Correspondence should be addressed to Changfan Zhang; zhangchangfan@263.net
}

Received 7 December 2016; Revised 28 January 2017; Accepted 5 February 2017; Published 9 March 2017

Academic Editor: Xiao He

Copyright (c) 2017 Jing He et al. This is an open access article distributed under the Creative Commons Attribution License, which permits unrestricted use, distribution, and reproduction in any medium, provided the original work is properly cited.

\begin{abstract}
This paper presents a fault-tolerant control scheme for a class of nonlinear systems with actuator faults and unknown input disturbances. First, the sliding mode control law is designed based on the reaching law method. Then, in view of unpredictable state variables and unknown information in the control law, the original system is transformed into two subsystems through a coordinate transformation. One subsystem only has actuator faults, and the other subsystem has both actuator faults and disturbances. A sliding mode observer is designed for the two subsystems, respectively, and the equivalence principle of the sliding mode variable structure is used to realize the accurate reconstruction of the actuator faults and disturbances. Finally, the observation value and the reconstruction value are used to carry out an online adjustment to the designed sliding mode control law, and fault-tolerant control of the system is realized. The simulation results are presented to demonstrate the approach.
\end{abstract}

\section{Introduction}

In recent years, theoretical research in fault-tolerant control has made great progress in the practical applications [18]. Fault-tolerant control scheme is widely studied in linear systems. Tao and $\mathrm{Xu}$ studied the fault-tolerant control of known and unknown parameters of high-speed train dynamics model $[9,10]$. Yu and Jiang proposed an innovative strategy for compensating the actuator faults to optimize system performance [11]. Shen et al. presented an integrated design method of adaptive robust control for a linear system with adaptive fault identification [12]. And Zhao et al. studied an adaptive sliding mode control for damage problems [13]. However, most of the actual objects are nonlinear, and some of the working points in the linear systems will enter the nonlinear region when it has a fault. For systems of actuator with random failures and uncertain parameters, Fan et al. studied its stabilization and tracking problem, but they did not consider the disturbance of unknown inputs [14]. Yin et al. presented a fault-tolerant control system scheme for realtime performance optimization, but they did not consider the effect of actuator faults [15]. In view of uncertain overdrive systems, Zhang et al. proposed a robust control allocation algorithm based on pseudoinverse, which is compensated for the negative influence of the failure and stuck fault [16]. Hu et al. presented an adaptive terminal sliding mode control method, found the finite time control of the attitude tracking, and solved the problem of actuator control input saturation [17]. However, these two methods did not obtain accurate fault values.

The concept of sliding mode variable structure control is to design the switching hyperplane of the system according to the expected dynamic characteristics of the system. The variable structure controller is used to drive the system state from the initial state to the switching hyperplane in a finite time and then maintain its state at the switching hyperplane. Once the system state reaches the switching hyperplane, the control function will ensure that the system travels along the switching hyperplane to reach the origin of the system. The system characteristics and parameters are entirely dependent on the designed switching hyperplane but are unrelated to external disturbances. Hence, the sliding mode variable structure control is extremely robust and has been widely applied in studies of nonlinear systems. 
Based on the work of Zhao et al. about the adaptive sliding mode control in the literature [13], considering a class of nonlinear systems with actuator faults and unknown input disturbances, this paper innovatively presents precisely a fault-tolerant control method with disturbance and fault reconstruction. To fulfill the above scheme, a fault-tolerant control law with sliding mode control is first proposed here. A fault diagnosis and reconstruction method is used to carry out accurate reconstruction of unknown information, and finally the observation value and the reconstruction value are used to carry out the corresponding adjustment of the control law. The simulation results show that the proposed control method can meet the requirements of control accurately and reliably.

The remainder of the paper is organized as follows. Section 2 describes the mathematical model of nonlinear systems with actuator faults and unknown input disturbances. Section 3 presents a fault-tolerant control law based on the sliding mode control. The design of observer is discussed in Section 4 . Then the fault reconstruction and the disturbance estimation are discussed in Section 5. Section 6 presents an online adjustment of the fault-tolerant control law. Simulation results are presented in Section 7, and conclusion is in Section 8 .

\section{Problem Description}

We have an uncertain nonlinear system affected by actuator faults and unknown disturbances:

$$
\begin{aligned}
& \dot{x}(t)=A x(t)+\Phi(x, u)+E f_{a}(t)+D d(t)+B u(t), \\
& y(t)=C x(t),
\end{aligned}
$$

where $x \in R^{n}$ is the state variables, $u \in R^{r}$ is the system inputs, and $y \in R^{p}$ is the outputs. Assume the nonlinear continuous term $\Phi(x, u) \in R^{n}$ is known. The unknown nonlinear term $d(t) \in R^{q}$ models the lumped uncertainties and disturbances experienced by the system, which is assumed to be bounded; that is, a positive constant $\gamma_{1}$ exists such that $\|d(t)\| \leq \gamma_{1}$. The unknown nonlinear term $f_{a}(t) \in R^{q}$ represents actuator faults, supposed to be norm bounded; that is, there exists a constant $\gamma_{2}$ such that $\left\|f_{a}(t)\right\| \leq \gamma_{2} . A \in R^{n \times n}, B \in R^{n \times r}$, $C \in R^{p \times n}, D \in R^{n \times q}$, and $E \in R^{n \times q}$ are known constant matrices with $n>p>q$.

\section{A Fault-Tolerant Control Law}

The active fault-tolerant control for faulty system (1) can be described as follows: If there is no fault, that is, $f_{a}(t)=0$, it is considered to be a nominal system; any appropriate control law is designed to achieve the gradual stability of the nominal system. If there is a fault, that is, $f_{a}(t) \neq 0$, an additional control law $u_{f}$ is designed to address and fix the fault. Thus, for a faulty system, the control law can be designed:

$$
u(t)=u_{n}(t)+u_{f}(t)
$$

where $u_{n}(t)$ is the nominal control law for the corresponding fault-free system.
If the specified system state command is defined as $r(t)$, $r(t) \in R^{n}$, the system error can be represented as

$$
e_{x}=r(t)-x(t) .
$$

Therefore, the sliding mode surface is represented as

$$
s_{0}=G e_{x}
$$

where $G$ is a matrix that will be designed.

From (4), the following equation can be obtained:

$$
\begin{aligned}
\dot{s}_{0}= & G \dot{e}_{x}=G(\dot{r}-\dot{x}) \\
= & G \dot{r} \\
& -G\left(A x(t)+\Phi(x, u)+E f_{a}(t)+D d(t)+B u(t)\right) .
\end{aligned}
$$

The control law is designed as

$$
\begin{aligned}
u= & (G B)^{*}\left(G \dot{r}-G A x-G \Phi(x, u)-G E f_{a}(t)\right. \\
& \left.-G D d(t)+k s_{0}+\varepsilon \operatorname{sgn}\left(s_{0}\right)\right),
\end{aligned}
$$

where parameters $k$ and $\varepsilon$ are constant to be designed and $(G B)^{*}$ is the generalized inverse matrix of $(G B)$. If there is no fault, that is, $f_{a}(t)=0$, the control law of the nominal system is

$$
\begin{aligned}
& u_{n}(t)=(G B)^{*}\left(G \dot{r}-G A x-G \Phi(x, u)-G D d(t)+k s_{0}\right. \\
& \left.\quad+\varepsilon \operatorname{sgn}\left(s_{0}\right)\right) .
\end{aligned}
$$

If there is a fault, that is, $f_{a}(t) \neq 0$, the additional control law $u_{f}$ is

$$
u_{f}(t)=(G B)^{*}\left(-G E f_{a}(t)\right) .
$$

Lemma 1. For the nonlinear system described in (1), the designed fault-tolerant control is shown in (6). When $k>0$ and $\varepsilon>0$, the system is in the stability condition of a sliding mode.

Proof. Consider the following Lyapunov function:

$$
V_{0}=\frac{1}{2} s_{0}^{2} \text {. }
$$

The time derivative of $V_{0}$, along with (5), is

$$
\begin{aligned}
\dot{V}_{0} & =s_{0} \dot{s}_{0}=s\left(G \dot{r}-G\left(A x(t)+\Phi(x, u)+E f_{a}(t)\right.\right. \\
& +D d(t)+B u(t))) .
\end{aligned}
$$

The following equation can be obtained by taking the substitution of (6) into (10):

$$
\dot{V}_{0}=-k s_{0}{ }^{2}-\varepsilon\left|s_{0}\right| .
$$

When $k>0$ and $\varepsilon>0$, the following equation can be obtained:

$$
\dot{V}_{0} \leq 0 \text {. }
$$

This completes the proof.

It can be seen from the above analysis that the system can satisfy the asymptotic stability requirement; namely, it will be driven to the corresponding sliding mode surface in a finite period of time. 


\section{Observer Design}

Equation (6) contains the system state variable $x$, the disturbance $d$, and the actuator fault $f_{a}$, where these variables are often unknown in control law. Therefore, we carry out the system state observations $x$ through the method of "designing observer," where the disturbance $d$ and actuator fault $f_{a}$ are reconstructed accurately again on this basis to obtain the corresponding state observation value $\hat{x}$ and reconstruction values $\widehat{d}$ and $\widehat{f}_{a}$ of the disturbance and fault, which are substituted into (6) to determine the control.

Assumption 2. $D$ is a column full rank matrix and $\operatorname{rank}(C D)=\operatorname{rank}(D)$.

Remark 3. If the disturbance distribution matrix $D$ is not a column full rank matrix, for example, $\operatorname{rank}(D)=q_{1}<q$, then a rank decomposition $D=D_{1}^{\prime} D_{2}^{\prime}$ could be applied, where $D_{1}^{\prime}$ is a column full rank matrix and $d_{1}^{\prime}(t)=D_{2}^{\prime} d_{2}^{\prime}(t)$ could be considered as a new unknown disturbance [18].

Assumption 4. The matrix pair $(A, C)$ is observable.

The matrix is partitioned for (1) to obtain the following equation:

$$
\begin{aligned}
\dot{x}(t)= & {\left[\begin{array}{l}
\dot{x}_{1}(t) \\
\dot{x}_{2}(t)
\end{array}\right] } \\
= & {\left[A_{11} A_{12} A_{21} A_{22}\right]\left[\begin{array}{l}
x_{1}(t) \\
x_{2}(t)
\end{array}\right]+\left[\begin{array}{l}
\Phi_{1}(x, u) \\
\Phi_{2}(x, u)
\end{array}\right] } \\
& +\left[\begin{array}{l}
E_{1} \\
E_{2}
\end{array}\right] f(t)+\left[\begin{array}{l}
D_{1} \\
D_{2}
\end{array}\right] d(t)+\left[\begin{array}{l}
B_{1} \\
B_{2}
\end{array}\right] u(t),
\end{aligned}
$$

where $x_{1}(t) \in R^{n-q}, x_{2}(t) \in R^{q}, A_{11} \in R^{(n-q) \times(n-q)}, A_{12} \in$ $R^{(n-q) \times q}, A_{21} \in R^{q \times(n-q)}, A_{22} \in R^{q \times q}, \Phi_{1}(x, u) \in R^{n-q}, \Phi_{2}(x$, $u) \in R^{q}, E_{1} \in R^{n-q}, E_{2} \in R^{q}, D_{1} \in R^{(n-q) \times q}$, and $D_{2} \in R^{q \times q}$ is a nonsingular matrix.

Based on Assumption 2, two transformation matrices, namely, $T$ and $S$, exist [19] such that

$$
x(t)=T^{-1} z(t)=T^{-1}\left[\begin{array}{l}
z_{1}(t) \\
z_{2}(t)
\end{array}\right] y(t)=S^{-1}\left[\begin{array}{l}
v_{1}(t) \\
v_{2}(t)
\end{array}\right] .
$$

Therefore, (1) could be converted as follows:

$$
\begin{aligned}
\dot{z}(t)= & {\left[\begin{array}{l}
\dot{z}_{1}(t) \\
\dot{z}_{2}(t)
\end{array}\right] } \\
= & T A T^{-1} z(t)+T \Phi(z, u)+T E f_{a}(t)+T D d(t) \\
& +T B u(t), \\
v(t)= & {\left[\begin{array}{l}
v_{1}(t) \\
v_{2}(t)
\end{array}\right]=S C T^{-1} z(t), }
\end{aligned}
$$

where

$$
S C T^{-1}=\left[\begin{array}{cc}
C_{11} & 0 \\
0 & C_{22}
\end{array}\right]
$$

and $C_{22}$ is a nonsingular matrix.

The following nonsingular transformation matrix $T$ is then constructed [19]:

$$
T=\left[I_{n-q}-D_{1} D_{2}^{-1} 0 I_{q}\right] .
$$

Therefore, the coefficient matrices in (15) are as follows:

$$
\begin{aligned}
T_{A}^{-1} & =\bar{A}=\left[\bar{A}_{11} \bar{A}_{12} \bar{A}_{21} \bar{A}_{22}\right], \\
T B & =\bar{B}=\left[\begin{array}{l}
\bar{B}_{1} \\
\bar{B}_{2}
\end{array}\right], \\
T D & =\bar{D}=\left[\begin{array}{c}
0 \\
\bar{D}_{2}
\end{array}\right], \\
T E & =\bar{E}=\left[\begin{array}{l}
\bar{E}_{1} \\
\bar{E}_{2}
\end{array}\right], \\
T \Phi(x, u) & =\bar{\Phi}(x, u)=\left[\begin{array}{l}
\bar{\Phi}_{1}(x, u) \\
\bar{\Phi}_{2}(x, u)
\end{array}\right],
\end{aligned}
$$

where $\bar{A}_{11}=A_{11}-D_{1} D_{2}^{-1} A_{21}, \bar{A}_{12}=\left(A_{11}-\right.$ $\left.D_{1} D_{2}^{-1} A_{21}\right) D_{1} D_{2}^{-1}+A_{12}-D_{1} D_{2}^{-1} A_{22}, \bar{A}_{21}=A_{21}, \bar{A}_{22}=$ $A_{21} D_{1} D_{2}^{-1}+A_{22}, \bar{\Phi}_{1}(x, u)=\Phi_{1}(x, u)-D_{1} D_{2}^{-1} \Phi_{2}(x, u)$, $\bar{\Phi}_{2}(x, u)=\Phi_{2}(x, u), \bar{E}_{1}=E_{1}-D_{1} D_{2}^{-1} E_{2}, \bar{E}_{2}=E_{2}, \bar{D}_{2}=D_{2}$, $\bar{B}_{1}=B_{1}-D_{1} D_{2}^{-1} B_{2}, \bar{B}_{2}=B_{2}, z_{1}(t) \in R^{n-q}, z_{2}(t) \in R^{q}$, $\bar{A}_{11} \in R^{(n-q) \times(n-q)}, \bar{A}_{12} \in R^{(n-q) \times q}, \bar{A}_{21} \in R^{q \times(n-q)}, \bar{A}_{22} \in R^{q \times q}$, $\bar{\Phi}_{1}(x, u) \in R^{n-q}, \bar{\Phi}_{2}(x, u) \in R^{q}, \bar{E}_{1} \in R^{n-q}, \bar{E}_{2} \in R^{q}, \bar{D}_{2} \in R^{q}$, $C_{11} \in R^{(p-q) \times(n-q)}, C_{22} \in R^{q \times q}, v_{1} \in R^{p-q}$, and $v_{2} \in R^{q}$.

System (15) is then transformed into the following subsystems:

$$
\begin{aligned}
\dot{z}_{1}(t)= & \bar{A}_{11} z_{1}(t)+\bar{A}_{12} z_{2}(t)+\bar{\Phi}_{1}(x, u)+\bar{E}_{1} f_{a}(t) \\
& +\bar{B}_{1} u(t), \\
v_{1}(t)= & C_{11} z_{1}(t), \\
\dot{z}_{2}(t)= & \bar{A}_{21} z_{1}(t)+\bar{A}_{22} z_{2}(t)+\bar{\Phi}_{2}(x, u)+\bar{E}_{2} f_{a}(t) \\
& +\bar{D}_{2} d(t)+\bar{B}_{2} u(t), \\
v_{2}(t)= & C_{22} z_{2}(t) .
\end{aligned}
$$

Two subsystems, (19) and (20), could be obtained from system (1) by matrix transformation. Subsystem (19) is free from any uncertainties but is subjected to system actuator faults; subsystem (20) has actuator faults and uncertainties. The detail design of two sliding mode observers corresponding to the above subsystems will be presented as follows.

Assumption 5. $\left(\bar{A}_{11}, C_{11}\right)$ and $\left(\bar{A}_{22}, C_{22}\right)$ are observable. 
Assumption 6. The nonlinear functions $\bar{\Phi}_{1}$ and $\bar{\Phi}_{2}$ are assumed to be known and satisfied the Lipschitz conditions, such that

$$
\begin{gathered}
\left\|\bar{\Phi}_{1}(x, u)-\bar{\Phi}_{1}(\widehat{x}, u)\right\| \leq \bar{\gamma}_{3}\|x-\widehat{x}\| \\
=\bar{\gamma}_{3}\left\|T^{-1}\right\|\|z-\widehat{z}\|=\gamma_{3}\|z-\widehat{z}\|, \\
\left\|\bar{\Phi}_{2}(x, u)-\bar{\Phi}_{2}(\widehat{x}, u)\right\| \leq \bar{\gamma}_{4}\|x-\hat{x}\| \\
=\bar{\gamma}_{4}\left\|T^{-1}\right\|\|z-\widehat{z}\|=\gamma_{4}\|z-\widehat{z}\|,
\end{gathered}
$$

where $\gamma_{3}$ and $\gamma_{4}$ are known Lipschitz positive constants.

Based on the transformed systems (19) and (20), the present study proposes the following two sliding mode observers:

$$
\begin{aligned}
\dot{\vec{z}}_{1}(t)= & \bar{A}_{11} \widehat{z}_{1}(t)+\bar{A}_{12} \widehat{z}_{2}(t)+\bar{\Phi}_{1}(\widehat{x}, u)+\bar{E}_{1} r_{1}(t) \\
& +\bar{B}_{1} u(t)+L_{1}\left(v_{1}(t)-\widehat{v}_{1}(t)\right), \\
\widehat{v}_{1}(t)= & C_{11} \widehat{z}_{1}(t), \\
\dot{\vec{z}}_{2}(t)= & \bar{A}_{21} \widehat{z}_{1}(t)+\bar{A}_{22} \widehat{z}_{2}(t)+\bar{\Phi}_{2}(\widehat{x}, u)+\bar{E}_{2} r_{2}(t) \\
& +\bar{B}_{2} u(t)+L_{2}\left(v_{2}(t)-\widehat{v}_{2}(t)\right), \\
\widehat{v}_{2}(t)= & C_{22} \widehat{z}_{2}(t),
\end{aligned}
$$

where superscript " " indicates an estimated value and $r_{1}(t)$ and $r_{2}(t)$ represent the input signals of the sliding mode observers:

$$
\begin{aligned}
& r_{1}(t)=\left\{-\rho_{1} \frac{F_{1}\left(\widehat{v}_{1}(t)-v_{1}(t)\right)}{\left\|F_{1}\left(\widehat{v}_{1}(t)-v_{1}(t)\right)\right\|} \text { if } \widehat{v}_{1}(t)-v_{1}(t)\right. \\
& \quad \neq 00 \text { if } \widehat{v}_{1}(t)-v_{1}(t)=0,
\end{aligned}
$$

$$
\begin{aligned}
& r_{2}(t)=\left\{-\rho_{2} \frac{F_{2}\left(\widehat{v}_{2}(t)-v_{2}(t)\right)}{\left\|F_{2}\left(\widehat{v}_{2}(t)-v_{2}(t)\right)\right\|} \text { if } \widehat{v}_{2}(t)-v_{2}(t)\right. \\
& \neq 00 \text { if } \widehat{v}_{2}(t)-v_{2}(t)=0,
\end{aligned}
$$

where $F_{1}$ and $F_{2}$ are the observer gains and $\rho_{1}$ and $\rho_{2}$ are positive scalars that will be designed.

Assumption 7. The existing arbitrary matrices $F_{1}$ and $F_{2}$ and symmetric positive definite matrices $P_{1}$ and $P_{2}$ will satisfy the following equations:

$$
\begin{aligned}
& P_{1} \bar{E}_{1}=C_{11}^{T} F_{1}^{T}, \\
& P_{2} \bar{E}_{2}=C_{22}^{T} F_{2}^{T} .
\end{aligned}
$$

From Assumption 5 we know that the existing matrices $L_{1}$ and $L_{2}$ will make $A_{10}$ and $A_{20}$ become stable matrices:

$$
\begin{aligned}
& \bar{A}_{11}-L_{1} C_{11}=A_{10}, \\
& \bar{A}_{22}-L_{2} C_{22}=A_{20} .
\end{aligned}
$$

If the state estimation errors are defined as $e_{1}=z_{1}(t)-\widehat{z}_{1}(t)$, $e_{2}=z_{2}(t)-\widehat{z}_{2}(t)$, and $e=\left(\begin{array}{ll}e_{1} & e_{2}\end{array}\right)^{T}$ and the output estimation errors are defined as $e_{v 1}=v_{1}(t)-\widehat{v}_{1}(t)=C_{11} e_{1}(t)$ and $e_{v 2}=$ $v_{2}(t)-\widehat{v}_{2}(t)=C_{22} e_{2}(t)$, then, from (19), (20), and (23), the state estimation errors dynamical systems are described by

$$
\begin{aligned}
\dot{e}_{1}(t)= & \left(\bar{A}_{11}-L_{1} C_{11}\right) e_{1}(t)+\bar{A}_{12} e_{2}(t)+\bar{\Phi}_{1}(z, u) \\
& -\bar{\Phi}_{2}(\widehat{z}, u)+\bar{E}_{1}\left(f_{a}(t)-r_{1}(t)\right), \\
\dot{e}_{2}(t)= & \left(\bar{A}_{22}-L_{2} C_{22}\right) e_{1}(t)+\bar{A}_{21} e_{1}(t)+\bar{\Phi}_{2}(z, u) \\
& -\bar{\Phi}_{2}(\widehat{z}, u)+\bar{E}_{2}\left(f_{a}(t)-r_{2}(t)\right) \\
& +\bar{D}_{2} d(t) .
\end{aligned}
$$

Lemma 8. Considering the error dynamics system (27) and (28) and Assumptions 6 and 7, if the following LMI is satisfied

$$
\left[\begin{array}{cccc}
A_{10}^{T} P_{1}+P_{1} A_{10}+\xi \gamma^{2} I_{n-q} & \bar{A}_{21}^{T} P_{2}+P_{1} \bar{A}_{12} & P_{1} & 0 \\
\bar{A}_{12}^{T} P_{1}+P_{2} \bar{A}_{21} & A_{20}^{T} P_{2}+P_{2} A_{20}+\xi \gamma^{2} I_{q} & 0 & P_{2} \\
P_{1} & 0 & -\xi I_{n-q} & 0 \\
0 & P_{2} & 0 & -\xi I_{q}
\end{array}\right]<0
$$

and the parameters $\rho_{1}$ and $\rho_{2}$ satisfy

$$
\begin{aligned}
& \rho_{1}>\gamma_{2}, \\
& \rho_{2}>\gamma_{4}+\frac{\left\|\bar{D}_{2}\right\|}{\left\|\bar{E}_{2}\right\|} \gamma_{1}
\end{aligned}
$$

then $e_{1}$ and $e_{2}$ are asymptotically convergent; that is,

$$
\begin{aligned}
& \lim _{t \rightarrow \infty} e_{1}(t)=0, \\
& \lim _{t \rightarrow \infty} e_{2}(t)=0,
\end{aligned}
$$

where $\xi$ is a positive constant and $I_{q}$ is a q-dimensional identity matrix. 
Proof. Consider the following Lyapunov function:

$$
V_{1}=e_{1}^{T} P_{1} e_{1}+e_{2}^{T} P_{2} e_{2}
$$

The derivative of $V$ along with the error dynamic systems (27) and (28) is

$$
\begin{aligned}
\dot{V}_{1} & =e_{1}^{T}\left(A_{10}^{T} P_{1}+P_{1} A_{10}\right) e_{1}+2 e_{1}^{T} P_{1}\left(\bar{A}_{12} e_{2}\right. \\
& \left.+\bar{\Phi}_{1}(z, u)-\bar{\Phi}_{1}(\widehat{z}, u)+\bar{E}_{1} f_{a}(t)-\bar{E}_{1} r_{1}(t)\right) \\
& +e_{2}^{T}\left(A_{20}^{T} P_{2}+P_{2} A_{20}\right) e_{2}+2 e_{2}^{T} P_{2}\left(\bar{A}_{21} e_{1}\right. \\
& +\bar{\Phi}_{2}(z, u)-\bar{\Phi}_{2}(\widehat{z}, u)+\bar{E}_{2} f_{a}(t)+\bar{D}_{2} d(t) \\
& \left.-\bar{E}_{2} r_{2}(t)\right) .
\end{aligned}
$$

Let

$$
\begin{aligned}
\bar{A} & =\left[\bar{A}_{11}-L_{1} C_{11} \bar{A}_{12} \bar{A}_{21} \bar{A}_{22}-L_{2} C_{22}\right], \\
P & =\left[P_{1} 00 P_{2}\right] ;
\end{aligned}
$$

then

$$
\begin{aligned}
\dot{V}_{1}= & e^{T}\left(\bar{A}^{T} P+P \bar{A}\right) e+2 e^{T} P(\bar{\Phi}(z, u)-\bar{\Phi}(\widehat{z}, u)) \\
& +2 e_{1}^{T} P_{1}\left(\bar{E}_{1} f_{a}(t)-\bar{E}_{1} r_{1}(t)\right) \\
& +2 e_{2}^{T} P_{2}\left(\bar{E}_{2} f_{a}(t)+\bar{D}_{2} d(t)-\bar{E}_{2} r_{2}(t)\right) .
\end{aligned}
$$

Since the inequality $2 X^{T} Y \leq(1 / \xi) X^{T} X+\xi Y^{T} Y$ is true for any scalar $\xi>0$, then

$$
\begin{array}{rl}
2 e^{T} & P(\bar{\Phi}(z, u)-\bar{\Phi}(\widehat{z}, u)) \\
& \leq \frac{1}{\xi} e^{T} P^{2} e
\end{array}
$$

$$
\begin{aligned}
& \quad+\xi(\bar{\Phi}(z, u)-\bar{\Phi}(\widehat{z}, u))^{T}(\bar{\Phi}(z, u)-\bar{\Phi}(\widehat{z}, u)) \\
& \leq \frac{1}{\xi} e^{T} P^{2} e+\xi \gamma^{2}\|e\|^{2} .
\end{aligned}
$$

From Assumption 7, the following equation can be obtained:

$$
\begin{aligned}
& 2 e_{1}^{T} P_{1} \bar{E}_{1} f_{a}(t)-2 e_{1}^{T} P_{1} \bar{E}_{1} r_{1}(t) \\
&= 2 e_{1}^{T} P_{1} \bar{E}_{1} f_{a}(t)-2 \rho_{1} e_{1}^{T} P_{1} \bar{E}_{1} \frac{F_{1} C_{11} e_{1}}{\left\|F_{1} C_{11} e_{1}\right\|} \\
& \leq 2\left\|F_{1} C_{11}\right\|\left\|e_{1}\right\|\left(\gamma_{2}-\rho_{1}\right) \leq 0, \\
& 2 e_{2}^{T} P_{2} \bar{E}_{2} f_{a}(t)+2 e_{2}^{T} P_{2} \bar{D}_{2} d(t)-2 e_{2}^{T} P_{2} \bar{E}_{2} r_{2}(t) \\
&=2 e_{2}^{T} P_{2} \bar{E}_{2} f_{a}(t)+2 e_{2}^{T} P_{2} \bar{D}_{2} d(t) \\
& \quad-2 \rho_{2} e_{2}^{T} P_{2} \bar{E}_{2} \frac{F_{2} C_{22} e_{2}}{\left\|F_{2} C_{22} e_{2}\right\|} \\
& \leq 2\left\|F_{2} C_{22}\right\|\left\|e_{2}\right\|\left(\gamma_{2}+\frac{\left\|\bar{D}_{2}\right\|}{\left\|\bar{E}_{2}\right\|} \gamma_{1}-\rho_{2}\right) \leq 0 .
\end{aligned}
$$

Equations (36) to (37) are substituted into (33) to obtain

$$
\dot{V}_{1} \leq e^{T}\left(\bar{A}^{T} P+P \bar{A}+\frac{1}{\xi} P^{2}+\xi \gamma^{2} I_{n}\right) e .
$$

$\dot{V}_{1}$ turns out to be negative definite by imposing

$$
\bar{A}^{T} P+P \bar{A}+\frac{1}{\xi} P^{2}+\xi \gamma^{2} I_{n}<0
$$

The linear matrix inequality is satisfied

$$
\left[\bar{A}^{T} P+P \bar{A}+\xi \gamma^{2} I_{n} P P-\xi I_{n}\right]<0
$$

such that

$$
\left[\begin{array}{cccc}
A_{10}^{T} P_{1}+P_{1} A_{10}+\xi \gamma^{2} I_{n-q} & \bar{A}_{21}^{T} P_{2}+P_{1} \bar{A}_{12} & P_{1} & 0 \\
\bar{A}_{12}^{T} P_{1}+P_{2} \bar{A}_{21} & A_{20}^{T} P_{2}+P_{2} A_{20}+\xi \gamma^{2} I_{q} & 0 & P_{2} \\
P_{1} & 0 & -\xi I_{n-q} & 0 \\
0 & P_{2} & 0 & -\xi I_{q}
\end{array}\right]<0
$$

so that $e(t)$ will make a global asymptotic convergence to zero; that is,

$$
\begin{aligned}
& \lim _{t \rightarrow \infty} e_{1}(t)=0, \\
& \lim _{t \rightarrow \infty} e_{2}(t)=0 .
\end{aligned}
$$

Remark 9. Lemma 8 implies that $e_{1}$ and $e_{2}$ are bounded; that is, $t_{0}$ will exist if $t>t_{0}$

$$
\begin{gathered}
\left\|e_{1}\right\| \leq \delta_{1}, \\
\left\|e_{2}\right\| \leq \delta_{2}, \\
\|e\| \leq \delta,
\end{gathered}
$$

where $\delta_{1}, \delta_{2}$, and $\delta$ are three finite positive scalars. 
Lemma 10. Choose the sliding mode surfaces $s_{1}=F_{1} e_{v 1}$ and $s_{2}=F_{2} e_{v 2}$. Suppose Assumptions 6 and 7 and inequality
(43) hold; besides, $\rho_{i}(i=1,2)$ is chosen sufficiently large to satisfy

$$
\begin{aligned}
& \rho_{1} \geq \frac{\left(\left\|\bar{A}_{11}-L_{1} C_{11}\right\|+\gamma_{3}\right) \delta_{1}+\left(\left\|\bar{A}_{12}\right\|+\gamma_{3}\right) \delta_{2}+\left\|\bar{E}_{1}\right\| \gamma_{2}+K_{1}}{\lambda_{\min }\left(\bar{E}_{1}^{T} P_{1} \bar{E}_{1}\right)}, \\
& \rho_{2} \geq \frac{\left\|F_{2} C_{22}\right\|\left(\left(\left\|\bar{A}_{22}-L_{2} C_{22}\right\|+\gamma_{4}\right) \delta_{2}+\left(\left\|\bar{A}_{21}\right\|+\gamma_{4}\right) \delta_{1}+\left\|\bar{E}_{2}\right\| \gamma_{2}+\left\|D_{2}\right\| \gamma_{1}\right)+K_{2}}{\lambda_{\min }\left(\bar{E}_{2}^{T} P_{2} \bar{E}_{2}\right)} .
\end{aligned}
$$

Then the error system (27) and (28) will be driven to the corresponding sliding mode surface $s_{i}=0(i=1,2)$ in finite time. $K_{1}$ and $K_{2}$ are positive constants.

Proof. (1) We select the Lyapunov function as follows:

$$
V_{2}=\frac{1}{2} s_{1}^{T} s_{1} .
$$

The time derivative of $V_{2}$ along the trajectories of system (27) is given by

$$
\begin{aligned}
\dot{V}_{2} & =s_{1}^{T}\left(F_{1} C_{11} \dot{e}_{1}\right)=s_{1}^{T} F_{1} C_{11}\left(\left(\bar{A}_{11}-L_{1} C_{11}\right) e_{1}(t)\right. \\
& +\bar{A}_{12} e_{2}(t)+\bar{\Phi}_{1}(z, u)-\bar{\Phi}_{1}(\widehat{z}, u)+\bar{E}_{1} f_{a}(t) \\
& \left.-\bar{E}_{1} r_{1}(t)\right)=s_{1}^{T}\left(F_{1} C_{11} \dot{e}_{1}\right) \\
& =s_{1}^{T} F_{1} C_{11}\left(\left(\bar{A}_{11}-L_{1} C_{11}\right) e_{1}(t)+\bar{A}_{12} e_{2}(t)\right. \\
& \left.+\bar{\Phi}_{1}(z, u)-\bar{\Phi}_{1}(\widehat{z}, u)+\bar{E}_{1} f_{a}(t)\right)-F_{1} C_{11} \bar{E}_{1} r_{1}(t) \\
& =s_{1}^{T} F_{1} C_{11}\left(\left(\bar{A}_{11}-L_{1} C_{11}\right) e_{1}(t)+\bar{A}_{12} e_{2}(t)\right. \\
& \left.+\bar{\Phi}_{1}(z, u)-\bar{\Phi}_{1}(\widehat{z}, u)+\bar{E}_{1} f_{a}(t)\right)-\rho_{1} s_{1}^{T} \bar{E}_{1}^{T} P_{1} \bar{E}_{1} \\
& +\frac{s_{1}}{\left\|s_{1}\right\|}
\end{aligned}
$$

From $\lambda_{\text {min }}\left(\bar{E}_{1}^{T} P_{1} \bar{E}_{1}\right)\left\|s_{1}\right\|^{2} \leq s_{1}^{T} \bar{E}_{1}^{T} P_{1} \bar{E}_{1} s_{1}$ and (43), it follows that

$$
\begin{aligned}
\dot{V}_{2} & \leq s_{1}^{T} F_{1} C_{11}\left(\left(\bar{A}_{11}-L_{1} C_{11}\right) e_{1}(t)+\bar{A}_{12} e_{2}(t)\right. \\
& \left.+\bar{\Phi}_{1}(z, u)-\bar{\Phi}_{1}(\widehat{z}, u)+\bar{E}_{1} f_{a}(t)\right) \\
& -\rho_{1} \lambda_{\min }\left(\bar{E}_{1}^{T} P_{1} \bar{E}_{1}\right)\left\|s_{1}\right\| \leq\left\|s_{1}\right\|\left(\left\|F_{1} C_{11}\right\|\right. \\
& \cdot\left(\left(\left\|\bar{A}_{11}-L_{1} C_{11}\right\|+\gamma_{3}\right) \delta_{1}+\left(\left\|\bar{A}_{12}\right\|+\gamma_{3}\right) \delta_{2}\right. \\
& \left.\left.+\left\|\bar{E}_{1}\right\| \gamma_{2}\right)-\rho_{1} \lambda_{\min }\left(\bar{E}_{1}^{T} P_{1} \bar{E}_{1}\right)\right) .
\end{aligned}
$$

We design $\rho_{1}$ which satisfies

$$
\geq \frac{\left(\left\|\bar{A}_{11}-L_{1} C_{11}\right\|+\gamma_{3}\right) \delta_{1}+\left(\left\|\bar{A}_{12}\right\|+\gamma_{3}\right) \delta_{2}+\left\|\bar{E}_{1}\right\| \gamma_{2}+K_{1}}{\lambda_{\min }\left(\bar{E}_{1}^{T} P_{1} \bar{E}_{1}\right)} ;
$$

then

$$
\dot{V}_{2} \leq-K_{1}\left\|s_{1}\right\| .
$$

(2) Consider a Lyapunov function candidate

$$
V_{3}=\frac{1}{2} s_{2}^{T} s_{2}
$$

The time derivative of $V_{3}$ along the trajectories of system (28) is given by

$$
\begin{aligned}
\dot{V}_{3} & =\left(F_{2} C_{22} e_{2}\right)^{T}\left(F_{2} C_{22} \dot{e}_{2}\right)=\left(F_{2} C_{22} e_{2}\right)^{T} \\
& \cdot F_{2} C_{22}\left(\left(\bar{A}_{22}-L_{2} C_{22}\right) e_{2}(t)+\bar{A}_{21} e_{1}(t)\right. \\
& +\bar{\Phi}_{2}(z, u)-\bar{\Phi}_{2}(\widehat{z}, u)+\bar{E}_{2} f_{a}(t)+D_{2} d(t) \\
& \left.-\bar{E}_{2} r_{2}(t)\right)=s_{2}^{T} F_{2} C_{22}\left(\left(\bar{A}_{22}-L_{2} C_{22}\right) e_{2}(t)\right. \\
& +\bar{A}_{21} e_{1}(t)+\bar{\Phi}_{2}(z, u)-\bar{\Phi}_{2}(\widehat{z}, u)+\bar{E}_{2} f_{a}(t) \\
& \left.+D_{2} d(t)\right)-\rho_{2} s_{2}^{T} \bar{E}_{2}^{T} P_{2} \bar{E}_{2} \frac{s_{2}}{\left\|s_{2}\right\|} .
\end{aligned}
$$

By the same reasoning as in the proof of the previous steps, it follows that

$$
\begin{aligned}
\dot{V}_{3} & \leq s_{2}^{T} F_{2} C_{22}\left(\left(\bar{A}_{22}-L_{2} C_{22}\right) e_{2}(t)+\bar{A}_{21} e_{1}(t)\right. \\
& \left.+\bar{\Phi}_{2}(z, u)-\bar{\Phi}_{2}(\widehat{z}, u)+\bar{E}_{2} f_{a}(t)+D_{2} d(t)\right) \\
& -\rho_{2} \lambda_{\min }\left(\bar{E}_{2}^{T} P_{2} \bar{E}_{2}\right)\left\|s_{2}\right\| \leq\left\|s_{2}\right\|\left(\left\|F_{2} C_{22}\right\|\right. \\
& \cdot\left(\left(\left\|\bar{A}_{22}-L_{2} C_{22}\right\|+\gamma_{4}\right) \delta_{2}+\left(\left\|\bar{A}_{21}\right\|+\gamma_{4}\right) \delta_{1}\right. \\
& \left.\left.+\left\|\bar{E}_{2}\right\| \gamma_{2}+\left\|D_{2}\right\| \gamma_{1}\right)-\rho_{2} \lambda_{\min }\left(\bar{E}_{2}^{T} P_{2} \bar{E}_{2}\right)\right) .
\end{aligned}
$$


We design $\rho_{2}$ which satisfies

$$
\rho_{2} \geq \frac{\left\|F_{2} C_{22}\right\|\left(\left(\left\|\bar{A}_{22}-L_{2} C_{22}\right\|+\gamma_{4}\right) \delta_{2}+\left(\left\|\bar{A}_{21}\right\|+\gamma_{4}\right) \delta_{1}+\left\|\bar{E}_{2}\right\| \gamma_{2}+\left\|D_{2}\right\| \gamma_{1}\right)+K_{2}}{\lambda_{\min }\left(\bar{E}_{2}^{T} P_{2} \bar{E}_{2}\right)} ;
$$

then

$$
\dot{V}_{3} \leq-K_{2}\left\|s_{2}\right\| .
$$

This completes the proof.

This result shows that the sliding mode reachability condition is satisfied. As a consequence, based on the sliding mode equivalent principle, an ideal sliding motion will take place on the surface $s_{i}=0(i=1,2)$ and after some finite time.

\section{Fault Reconstruction and Disturbance Estimation}

When the system reaches the sliding mode surface, $s_{i}=\dot{s}_{i}=$ $0(i=1,2)$ according to the sliding mode equivalent principle [20]. The following equations are obtained:

$$
\begin{aligned}
& F_{1} e_{v 1}=F_{1} \dot{e}_{v 1}=F_{1} C_{11} \dot{e}_{1}=0, \\
& F_{2} e_{v 2}=F_{2} \dot{e}_{v 2}=F_{2} C_{22} \dot{e}_{2}=0 .
\end{aligned}
$$

5.1. The Reconstruction of Actuator Fault $f_{a}(t)$. From (55), we obtain the following:

$$
\begin{gathered}
F_{1} C_{11}\left(\left(\bar{A}_{11}-L_{1} C_{11}\right) e_{1}(t)+\bar{A}_{12} e_{2}(t)+\bar{\Phi}_{1}(z, u)\right. \\
\left.-\bar{\Phi}_{1}(\widehat{z}, u)+\bar{E}_{1} f_{a}(t)-\bar{E}_{1} r_{1}(t)\right)=0 .
\end{gathered}
$$

From (42), it follows that

$$
f_{a}(t) \approx r_{1}(t)=\rho_{1} \operatorname{sgn}\left(F_{1} e_{v 1}\right) .
$$

The sigmoid function was used to replace $\operatorname{sgn}(s)$ to weaken the chattering problem of the sliding mode, where the reconstructed value of the actuator fault is

$$
\hat{f}_{a}=\rho_{1}\left(\frac{2}{1+e^{-a_{1} F_{1} e_{v 1}}}-1\right),
$$

where $a_{1}$ is a positive constant to be designed.

5.2. The Estimation of the Unknown Input Disturbance $d(t)$. From (56), we obtain the following:

$$
\begin{array}{r}
F_{2} C_{22}\left(\left(\bar{A}_{22}-L_{2} \bar{C}_{22}\right) e_{2}(t)+\bar{A}_{21} e_{1}(t)+\bar{\Phi}_{2}(x, u)\right. \\
\left.-\bar{\Phi}_{2}(\hat{x}, u)+\bar{E}_{2} f_{a}(t)-\bar{E}_{2} r_{2}(t)+\bar{D}_{2} d(t)\right)=0 .
\end{array}
$$

From (42), it follows that

$$
\begin{aligned}
d(t) & \approx \bar{D}_{2}^{-1}\left(\bar{E}_{2} r_{2}(t)-\bar{E}_{2} f_{a}(t)\right) \\
& =\bar{D}_{2}^{-1} \bar{E}_{2}\left(\rho_{2} \operatorname{sgn}\left(F_{2} e_{v 2}\right)-\rho_{1} \operatorname{sgn}\left(F_{1} e_{v 1}\right)\right) .
\end{aligned}
$$

The sigmoid function was used to replace $\operatorname{sgn}(s)$ to weaken the chattering problem of the sliding mode, where the reconstruction value of the disturbance is

$$
\begin{aligned}
\widehat{d}(t) & =\bar{D}_{2}^{-1} \bar{E}_{2}\left(\rho_{2}\left(\frac{2}{1+e^{-a_{2} F_{2} e_{\nu 2}}}-1\right)\right. \\
- & \left.\rho_{1}\left(\frac{2}{1+e^{-a_{1} F_{1} e_{\nu 1}}}-1\right)\right),
\end{aligned}
$$

where $a_{2}$ is a positive constant to be designed.

\section{Adjustment of the Fault-Tolerant Control Law}

In view of the control law $u$ designed for the faulted system in (1), the observed value $\hat{x}$ of the system state, the reconstructed values $\widehat{d}$ from (62), and $\hat{f}_{a}$ from (59) of the disturbance and fault, respectively, were substituted into (6). The adjusted control law becomes

$$
\begin{aligned}
u= & (G B)^{*}\left(G \dot{r}-G A \hat{x}-G \Phi(\widehat{x}, u)-G E \hat{f}_{a}(t)\right. \\
& \left.-G D \hat{d}(t)+k s_{0}+\varepsilon \operatorname{sgn}\left(s_{0}\right)\right) .
\end{aligned}
$$

At this point, the control law in (7) adjusted to a nominal system is

$$
\begin{aligned}
& u_{n}(t)=(G B)^{*}\left(G \dot{r}-G A \hat{x}-G \Phi(\widehat{x}, u)+k s_{0}\right. \\
& \left.\quad+\varepsilon \operatorname{sgn}\left(s_{0}\right)-G D \hat{d}(t)\right) .
\end{aligned}
$$

The additional control law in (8) is adjusted to

$$
u_{f}(t)=(G B)\left(-G E \widehat{f}_{a}(t)\right) .
$$

Stability analysis is as follows.

Consider the following Lyapunov function:

$$
V_{4}=\frac{1}{2} s_{0}^{2} .
$$

The time derivative of $V_{4}$ along with (5) and (63) is

$$
\begin{aligned}
\dot{V}_{4} & =s_{0} \dot{s}_{0}=s_{0}\left(G \dot{r}-G A x-G D d-G E f_{a}\right. \\
& -G \Phi(x, u)-G B(G B)^{*}(G \dot{r}-G A \widehat{x}-G \Phi(\widehat{x}, u) \\
& \left.\left.-G E \widehat{f}_{a}(t)-G D \widehat{d}(t)+k s_{0}+\varepsilon \operatorname{sgn}\left(s_{0}\right)\right)\right) \\
& =s_{0}\left[-k s_{0}-\varepsilon \operatorname{sgn}\left(s_{0}\right)-G A(x-\widehat{x})-G(\Phi(x, u)\right. \\
& \left.-\Phi(\widehat{x}, u))-G E\left(f_{a}-\widehat{f}_{a}\right)-G D(d-\widehat{d})\right]
\end{aligned}
$$




$$
\begin{aligned}
& =s_{0}\left[-k s_{0}-\varepsilon \operatorname{sgn}\left(s_{0}\right)-G A T^{-1} e-G(\Phi(x, u)\right. \\
& \left.-\Phi(\widehat{x}, u))-G E\left(f_{a}-\widehat{f}_{a}\right)-G D(d-\widehat{d})\right] .
\end{aligned}
$$

It follows from (43) that

$$
\begin{aligned}
\dot{V}_{4} & \leq-k s_{0}{ }^{2}-\varepsilon\left|s_{0}\right|+\left|s_{0}\right|\left(\left\|G A T^{-1} \delta\right\|\right. \\
& \left.+\left\|G D \bar{D}_{2}^{-1} \bar{E}_{2}\left(\rho_{2}+\rho_{1}\right)\right\|+\left\|G E \rho_{1}\right\|+\|G \gamma \delta\|\right) .
\end{aligned}
$$

Finally, if the positive constant $\varepsilon$ is chosen such that

$$
\begin{aligned}
\varepsilon \geq & \delta\left\|G A T^{-1}+\left(\rho_{2}+\rho_{1}\right)\right\|\left\|G D \bar{D}_{2}^{-1} \bar{E}_{2}\right\|+\rho_{1}\|G E\| \\
& +\gamma \delta\|G\|
\end{aligned}
$$

we obtain

$$
\dot{V}_{4} \leq-k s_{0}^{2}
$$

It can be seen from the above analysis that the system can satisfy the asymptotic stability.

\section{Simulation Example}

Consider a robot arm whose motion equation is as follows [21]:

$$
\begin{aligned}
& M(q) \ddot{q}+B(q, \dot{q}) \dot{q}+G(q)=u, \\
& M(q)=\left[J_{1} 00 J_{m}\right], \\
& B(q, \dot{q})=\left[F_{n} 00 F_{m}\right], \\
& G(q)=\left[m g l \sin \left(q_{1}\right)+a\left(q_{1}-q_{2}\right)-a\left(q_{1}-q_{2}\right)\right],
\end{aligned}
$$

where $q_{1}$ and $q_{2}$ are the link displacement and the rotor displacement, respectively. The link inertia $J_{1}$, motor rotor inertia $J_{m}$, elastic constant $a$, link mass $m$, gravity $g$, connecting rod length $l$, and viscous friction coefficients $F_{n}$ and $F_{m}$ are all positive constant parameters. The control $u$ is the torque delivered by the motor. The robot parameters are $F_{n}=$ $0.5 \mathrm{Nm} /(\mathrm{rad} / \mathrm{s}), J_{m}=1 \mathrm{Nm}^{2}, k=2 \mathrm{Nm} / \mathrm{rad}, F_{m}=1, J_{1}=$ $2 \mathrm{Nm}^{2}, m=0.15 \mathrm{~kg}, g=9.8 \mathrm{~m} / \mathrm{s}^{2}$, and $l=0.3 \mathrm{~m}$.

From (1), the parameter matrixes are

$$
A=\left[\begin{array}{cccc}
0 & 1 & 0 & 0 \\
\frac{-k}{J_{1}} & \frac{-F_{1}}{J_{1}} & \frac{k}{J_{1}} & 0 \\
0 & 0 & 0 & 1 \\
\frac{k}{J_{m}} & 0 & \frac{-k}{J_{m}} & \frac{-F_{m}}{J_{m}}
\end{array}\right]
$$

$$
=\left[\begin{array}{cccc}
0 & 1 & 0 & 0 \\
-1 & -0.25 & 1 & 0 \\
0 & 0 & 0 & 1 \\
2 & 0 & -2 & -1
\end{array}\right]
$$$$
f(x, u, t)=\left[\begin{array}{c}
0 \\
-0.2205 \sin x_{1} \\
0 \\
0
\end{array}\right] \text {, }
$$$$
E=[00240021]
$$$$
D=[10001002] \text {, }
$$$$
B=[00100001] \text {, }
$$

$$
C=\left[\begin{array}{llll}
1 & 0 & 0 & 0 \\
0 & 0 & 1 & 0 \\
0 & 1 & 0 & 1 \\
0 & 0 & 0 & 1
\end{array}\right] \text {. }
$$

The transformational matrix $T$ is

$$
T=\left[\begin{array}{cccc}
1 & 0 & -1 & 0 \\
0 & 1 & 0 & 0 \\
0 & 0 & 1 & 0 \\
0 & 0 & 0 & 1
\end{array}\right] .
$$

The transformational matrix $S$ is

$$
S=\left[\begin{array}{cccc}
1 & -1 & 0 & 0 \\
0 & 0 & 1 & -1 \\
0 & 1 & 0 & 0 \\
0 & 0 & 0 & 1
\end{array}\right] .
$$

Set the matrices as

$$
\begin{aligned}
& P_{1}=[2001], \\
& P_{2}=[1001], \\
& F_{1}=[0204], \\
& F_{2}=[0201], \\
& L_{1}=[3-123.25], \\
& L_{2}=[4-12-1], \\
& G=\left[\begin{array}{llll}
5 & 1 & 0 & 0 \\
0 & 0 & 5 & 1
\end{array}\right] .
\end{aligned}
$$

In the control law of (63), the constants are taken to be $k=5$ and $\varepsilon=2$. The robotic arm system (71) is a dual input system, where the input signal 1 is $r_{1}=2 \cos (\pi t)$ and the input 


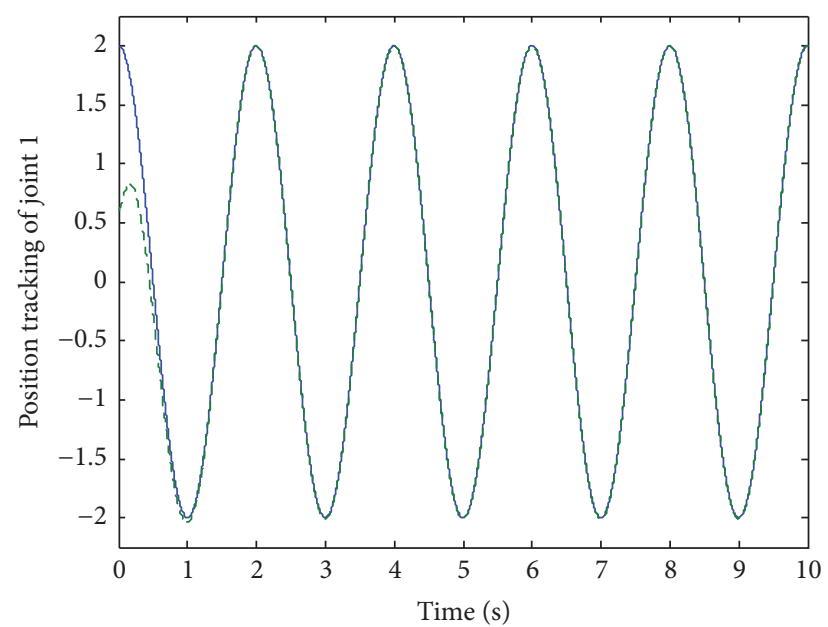

$-r_{1}$

$--x_{1}$

FIGURE 1: Actual value $x_{1}$ and tracking state of input signal 1.

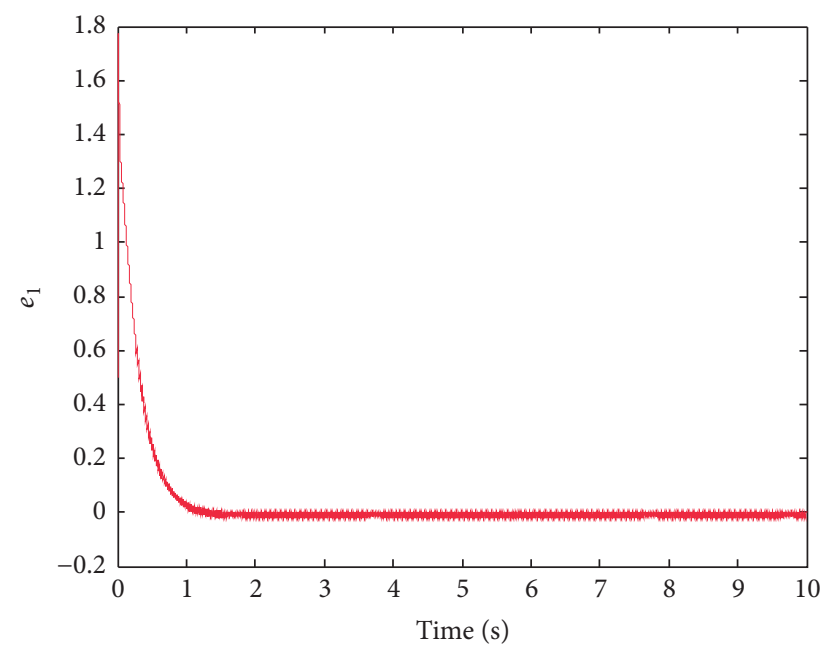

FIGURE 2: Tracking error $e_{1}$ of input signal 1.

signal 2 is $r_{2}=5 \sin (\pi t)$. Two square signals were chosen as the "unknown" input disturbance, where one had a period of $1 \mathrm{~s}$ and an amplitude of 1 and the other had a period of $2 \mathrm{~s}$ with an amplitude of 2 . The initial values of the system state variable $x(t)$ were taken as $0.6,0.3,-1.5$, and 0.5 , respectively. For faultless systems, the simulated experimental results are as follows.

Figures 1 and 3 show two states of $x_{1}$ and $x_{3}$ used to separately track the two input signals in the case of different initial values; the given input values were reached quickly, and they remained stable. The errors, $e_{1}$ and $e_{2}$, shown in Figures 2 and 4 , prove the point that the tracking effect is good. Figures 5 and 6 are two output signals of the control law, respectively.

When a system contains an actuator fault, a sinusoidal signal and a cosine signal were chosen to, respectively, simulate the fault: for example, $f_{a 1}(t)=\sin (5 t)$ and $f_{a 2}(t)=\cos (5 t)$.

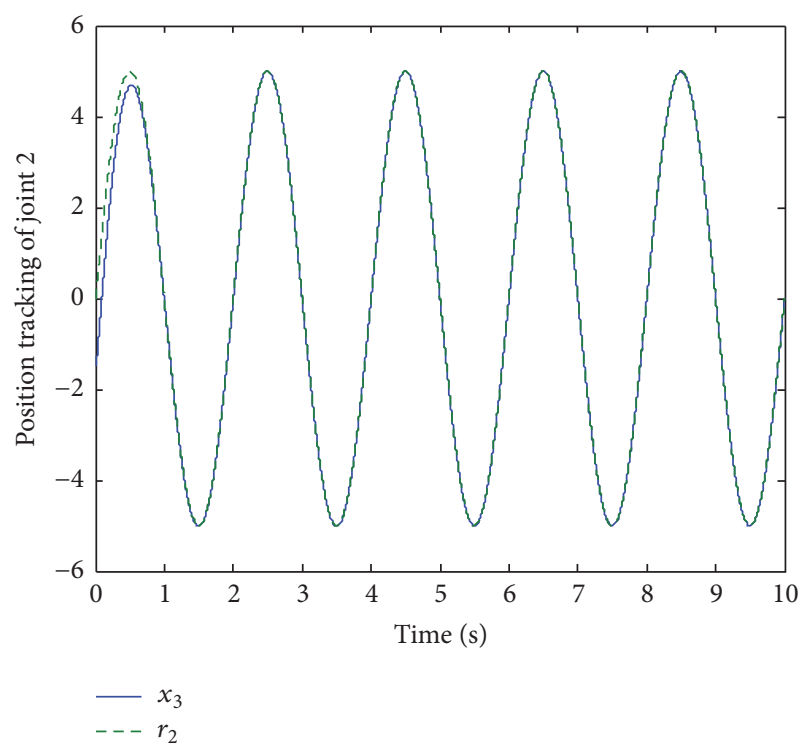

FIgURE 3: Actual value $x_{3}$ and tracking state of input signal 2 .

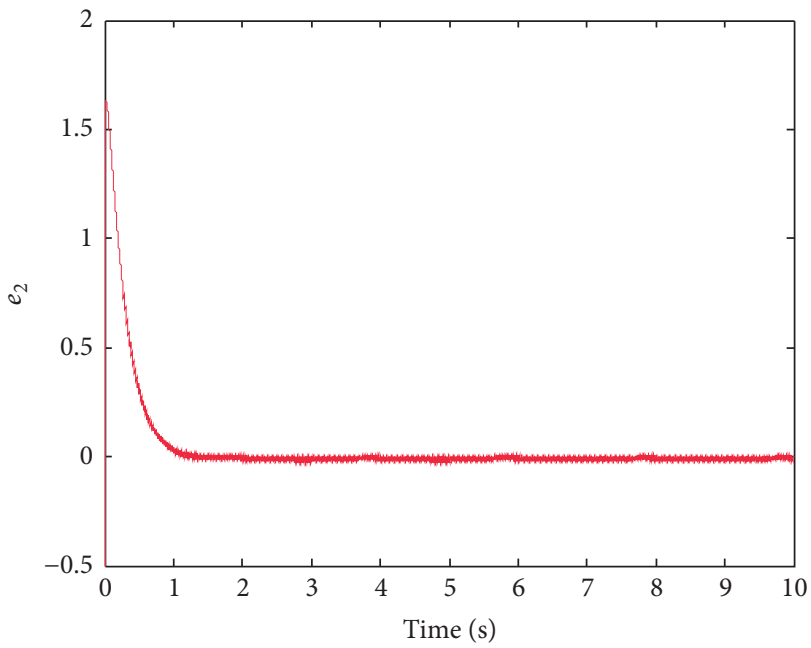

FIGURE 4: Tracking error $e_{2}$ of input signal 2.

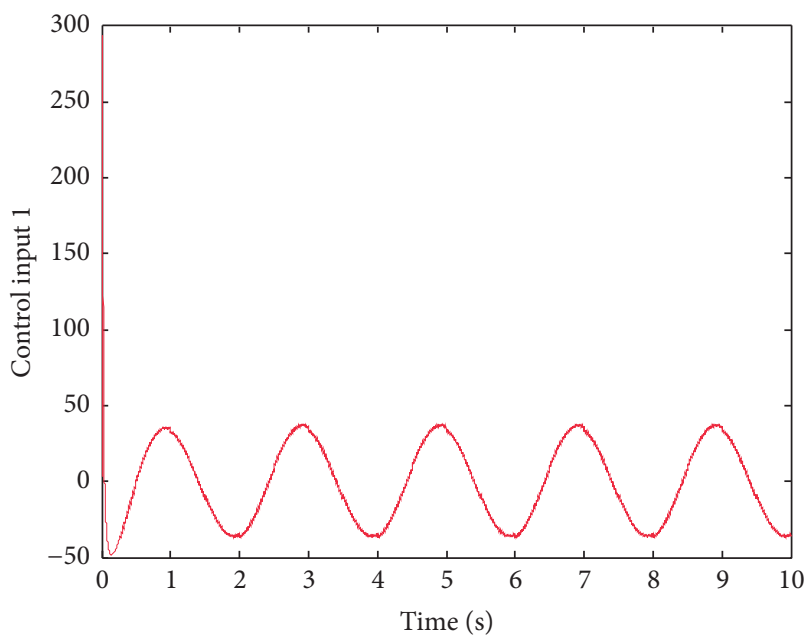

FIgURE 5: Control input signal $u_{1}$. 


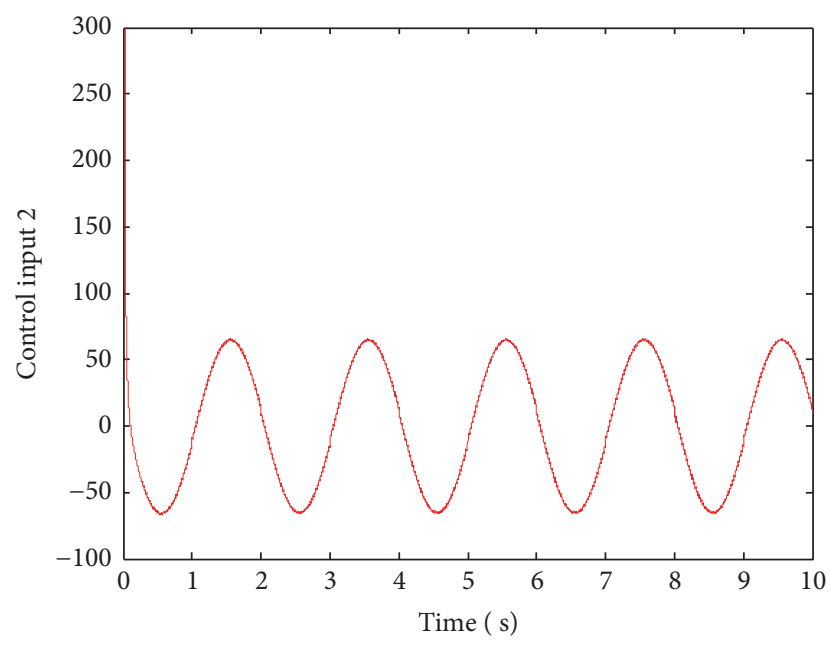

FIgURE 6: Control input signal $u_{2}$.

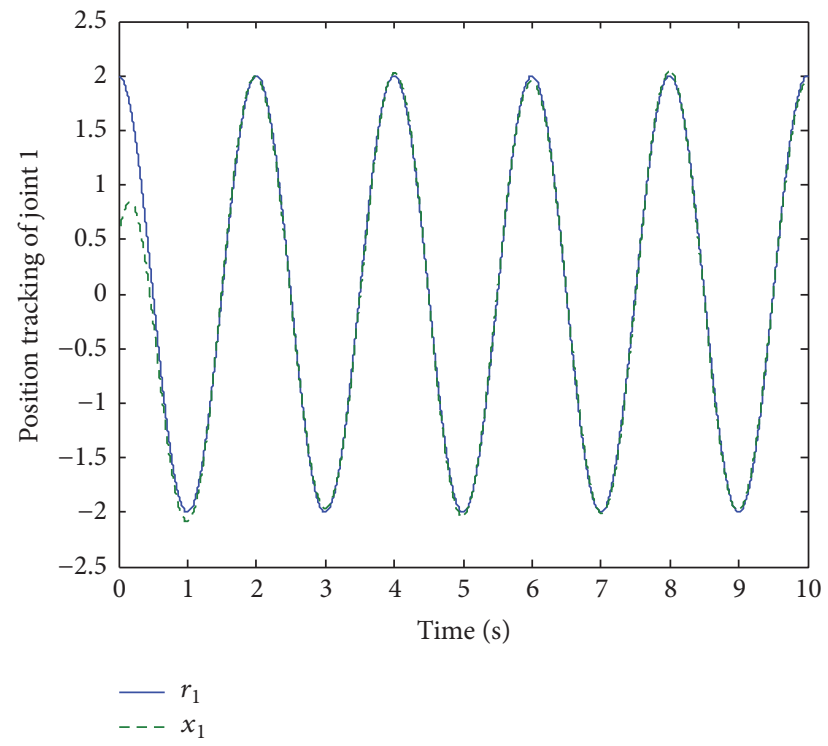

FIGURE 7: Actual value $x_{1}$ and tracking state of input signal 1 .

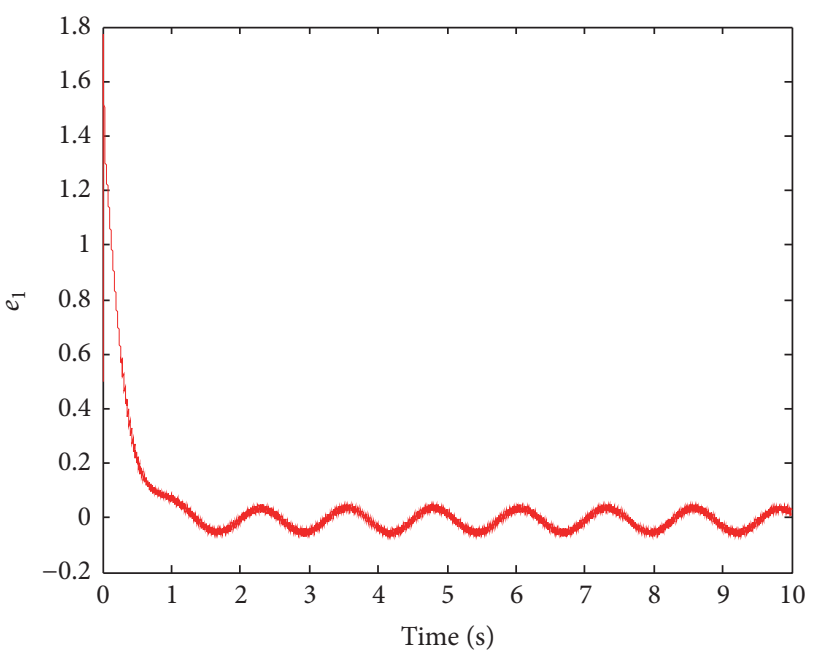

FIGURE 8: Tracking error $e_{1}$ of input signal 1.

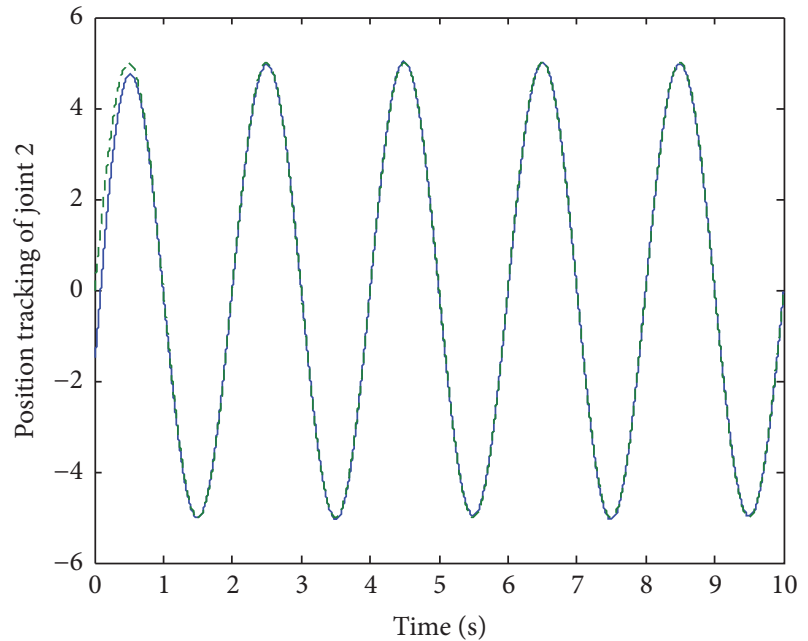

$-x_{3}$

FIGURE 9: Actual value $x_{3}$ and tracking state of input signal 2.

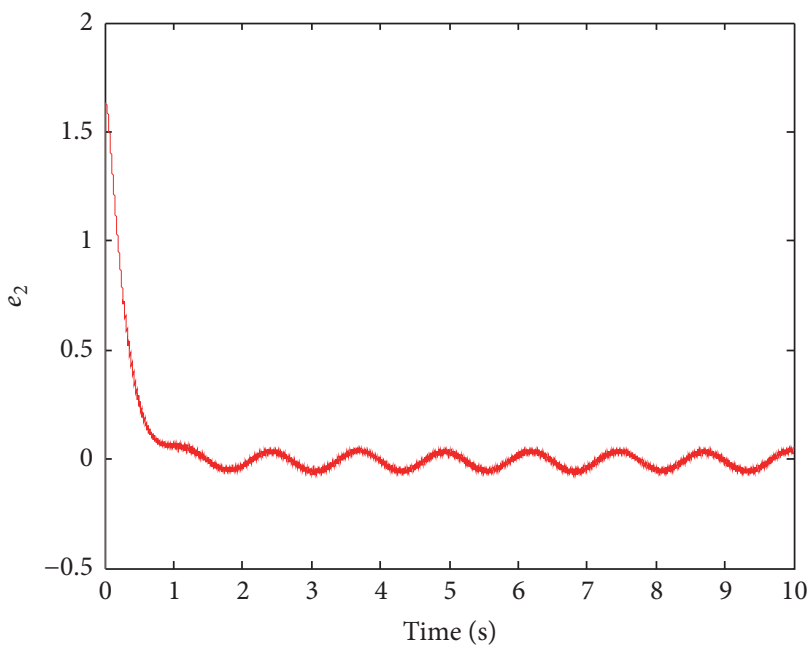

FIGURE 10: Tracking error $e_{2}$ of input signal 2.

Thus the fault-tolerant control was not carried out; namely, the additional control law $u_{f}$ was not added at this point. Figures 7 and 9 show the result of two input signals tracking, and Figures 8 and 10 show the result of two tracking errors.

It can be seen from Figures 7, 8, 9, and 10 that the input signals cannot be accurately tracked, and the tracking errors converge to 0 . When the additional control law $u_{f}$ is added for fault-tolerant control, the input signal tracking results are shown in Figures 15 and 17, and the results of two tracking errors are shown in Figures 16 and 18. The reconstruction results are shown in Figures 11, 12, 13, and 14.

Figures 11 and 12 display the unknown disturbances $d_{1}$ and $d_{2}$ and their estimated values, respectively. Meanwhile, Figures 13 and 14 show the actuator faults $f_{a 1}$ and $f_{a 2}$ and their reconstruction values separately. The simulation 


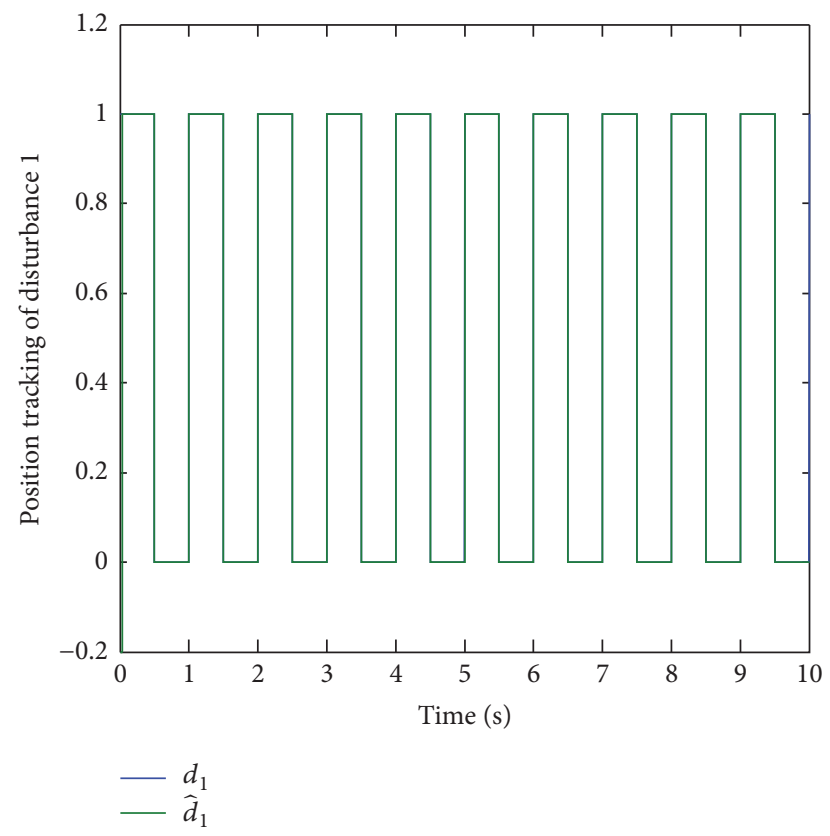

FIGURE 11: Unknown input disturbance $d_{1}$ and its estimation value.

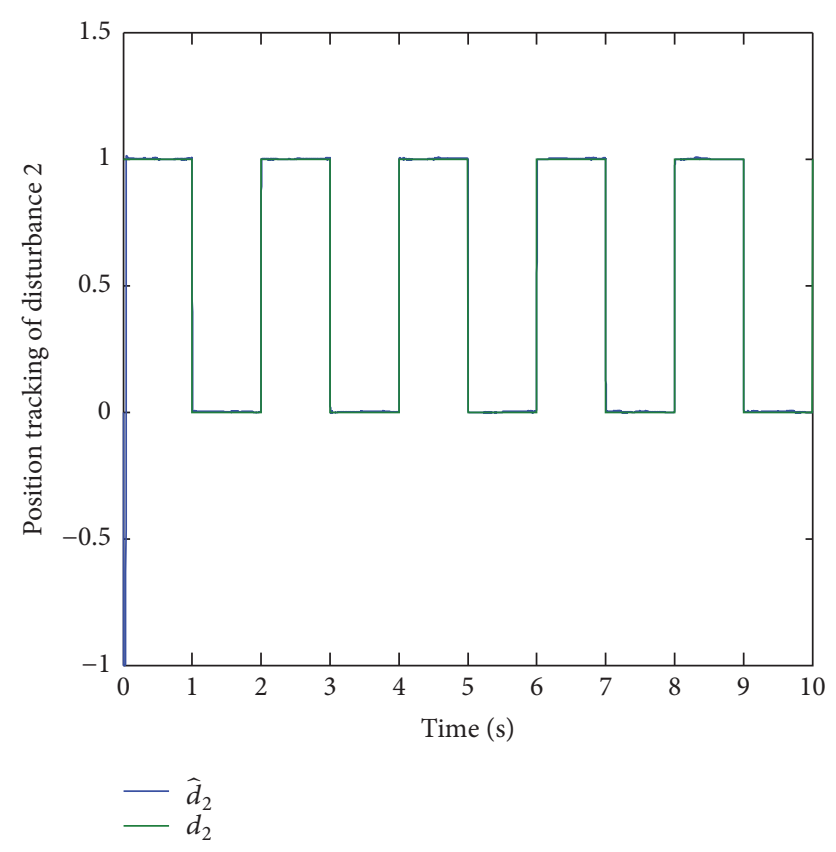

FIGURE 12: Unknown input disturbance $d_{2}$ and its estimation value.

results indicate that the method can estimate the unknown disturbances and reconstruct the actuator faults accurately. And the tracking effect is good.

Compared with Figures 7, 8, 9, and 10, it can be seen from Figures 15, 16, 17, and 18 that the input signals can be accurately tracked after the additional control law $u_{f}$ is added, and the tracking errors can converge to 0 . Figures 19 and 20 are two output signals of the control law, respectively.

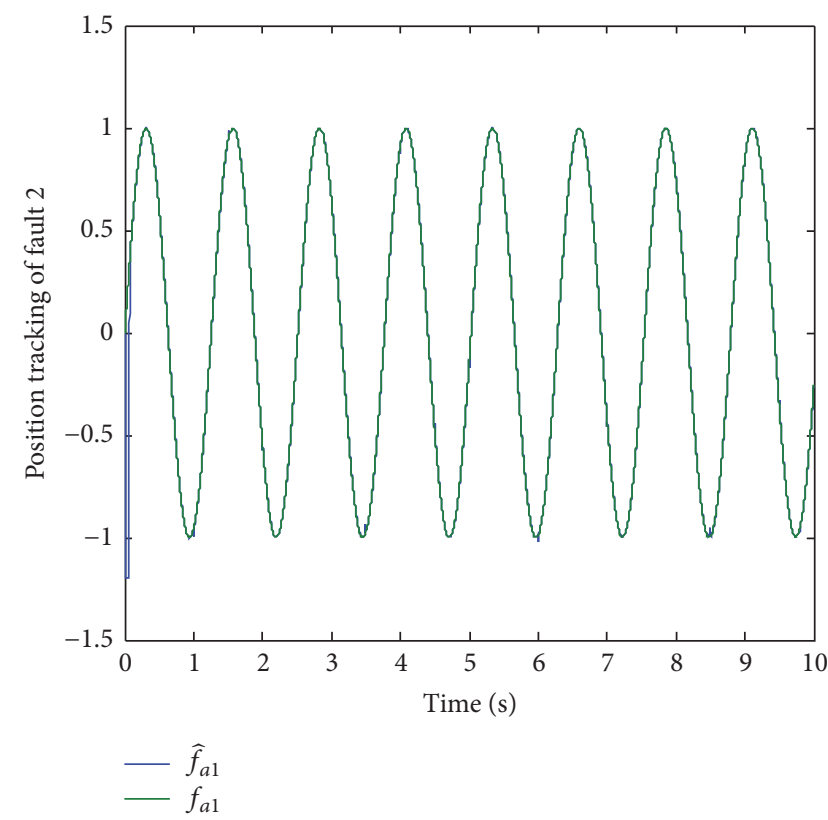

FIGURE 13: Actuator fault $f_{a 1}$ and its reconstruction value.

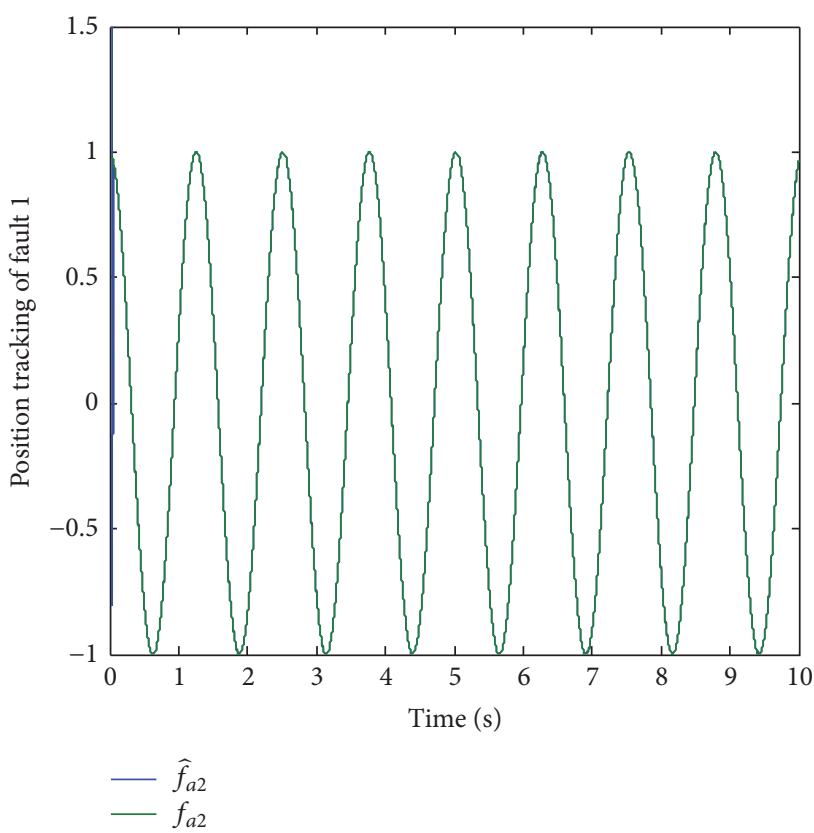

FIGURE 14: Actuator fault $f_{a 2}$ and its reconstruction value.

\section{Conclusions}

In this paper, we proposed a fault-tolerant control scheme based on fault reconstruction for a class of nonlinear systems with actuator faults and unknown input disturbances. It can obtain information from the unknown system state and can restrain the influence of the fault and disturbance. Therefore, this method has very strong engineering practicability. From the simulated experimental results applied on a mechanical 


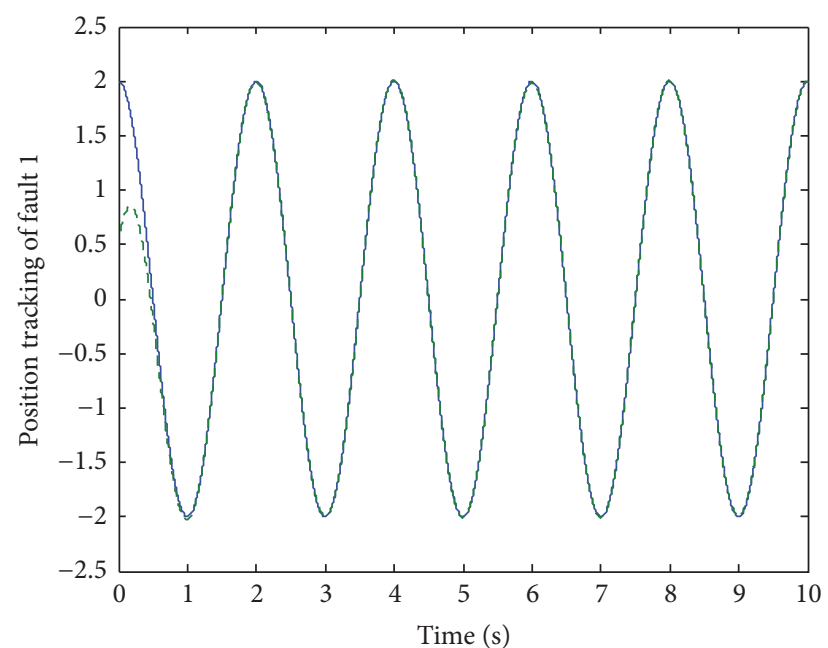

$-r_{1}$

Figure 15: Actual value $x_{1}$ and tracking state of input signal 1 .

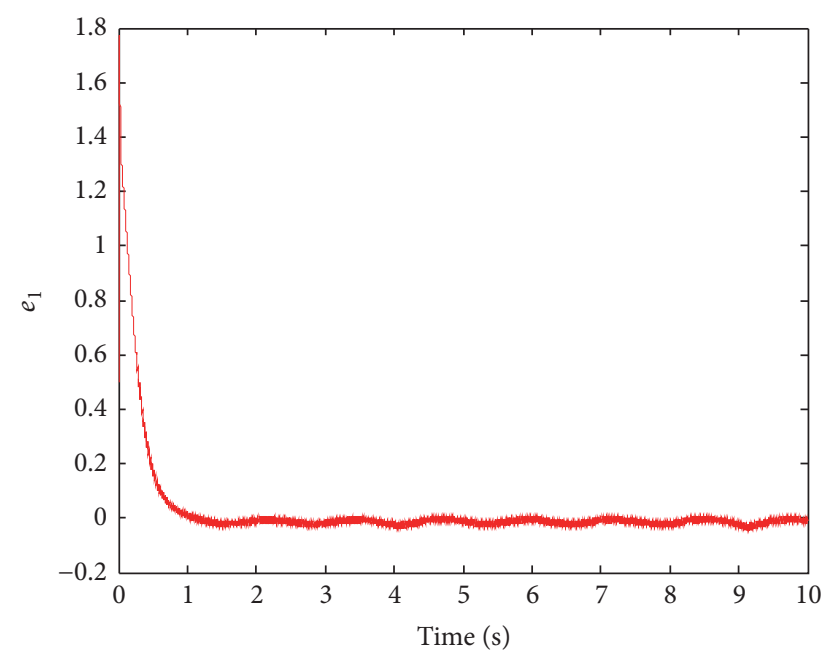

FIGURE 16: Tracking error $e_{1}$ of input signal 1.

are, our method can maintain the system asymptotic stability and can achieve a precise control purpose when it contains an actuator fault and an unknown input disturbance, which demonstrates that our proposed method provides a new way to control nonlinear systems with actuator faults and unknown disturbances in practical engineering applications.

\section{Competing Interests}

The authors declare that there is no conflict of interests regarding the publication of this paper.

\section{Acknowledgments}

This work was supported by the Natural Science Foundation of China (nos. 61473117 and 61273157) and Hunan Provincial

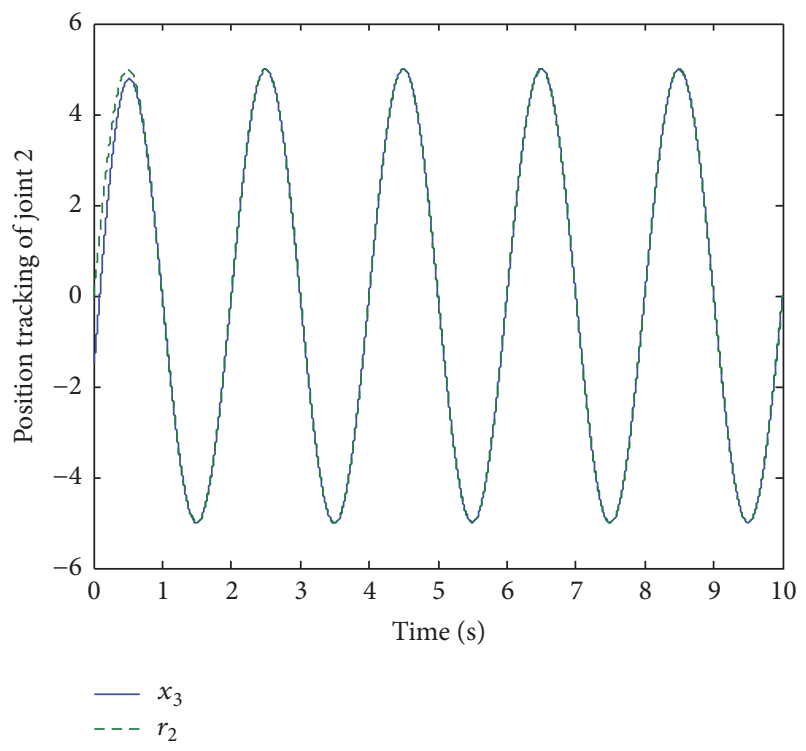

Figure 17: Actual value $x_{3}$ and tracking state of input signal 2 .

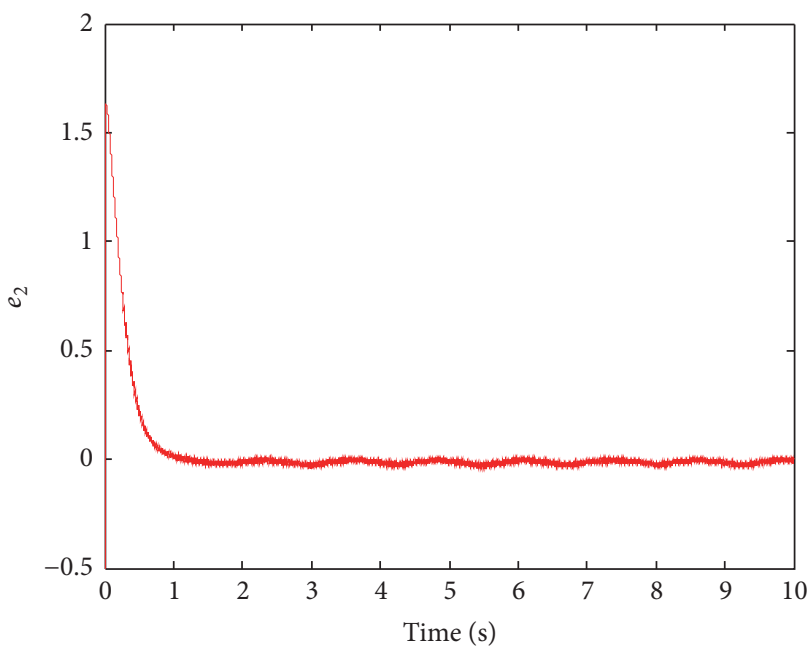

FIGURE 18: Tracking error $e_{2}$ of input signal 2.

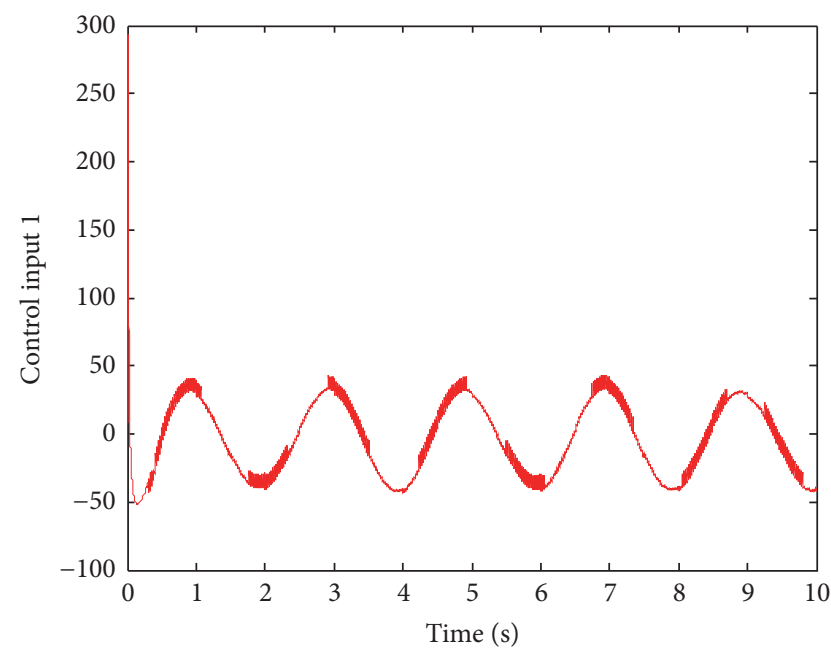

Figure 19: Control input signal $u_{1}$. 


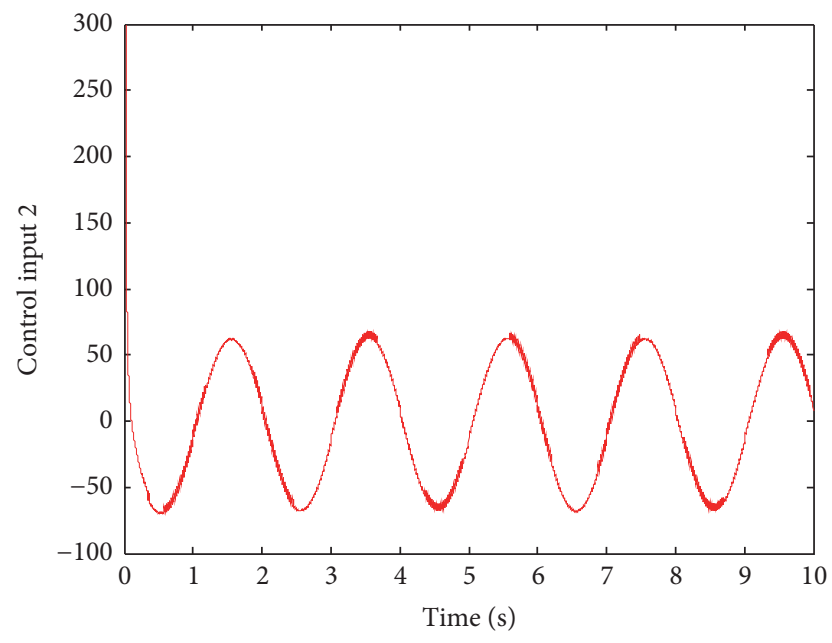

Figure 20: Control input signal $u_{2}$.

Natural Science Foundation of China (nos. 2016JJ5007 and 2015JJ5011).

\section{References}

[1] B. Mogens, K. Michel, L. Jan, and S. Marcel, Diagnosis and FaultTolerant Control, Springer, Berlin, Germany, 2nd edition, 2006.

[2] Z. Zhou, C. Hu, H. Fan, and J. Li, "Fault prediction of the nonlinear systems with uncertainty," Simulation Modelling Practice and Theory, vol. 16, no. 6, pp. 690-703, 2008.

[3] X. He, Z. Wang, and D. H. Zhou, "Network-based robust fault detection with incomplete measurements," International Journal of Adaptive Control and Signal Processing, vol. 23, no. 8, pp. 737-756, 2009.

[4] M. A. Djeziri, R. Merzouki, B. O. Bouamama, and M. Ouladsine, "Fault diagnosis and fault-tolerant control of an electric vehicle overactuated," IEEE Transactions on Vehicular Technology, vol. 62, no. 3, pp. 986-994, 2013.

[5] R. Loureiro, S. Benmoussa, Y. Touati, R. Merzouki, and B. Ould Bouamama, "Integration of fault diagnosis and fault-tolerant control for health monitoring of a class of MIMO intelligent autonomous vehicles," IEEE Transactions on Vehicular Technology, vol. 63, no. 1, pp. 30-39, 2014.

[6] D. Zhang, W.-A. Zhang, L. Yu, and Q.-G. Wang, "Distributed fault detection for a class of large-scale systems with multiple incomplete measurements," Journal of the Franklin Institute, vol. 352, no. 9, pp. 3730-3749, 2015.

[7] D. Zhang, P. Shi, W. Zhang, and L. Yu, "Energy-efficient distributed filtering in sensor networks: a unified switched system approach," IEEE Transactions on Cybernetics, pp. 1-12, 2016.

[8] X. He, Z. D. Wang, L. G. Qin, and D. H. Zhou, "Active faulttolerant control for an internet-based networked three-tank system," IEEE Transactions on Control Systems Technology, vol. 24, pp. 2150-2157, 2016.

[9] T. Tao and H. Xu, "Fault-tolerant control of high-speed trains with uncertain parameters and actuator failures using control reallocation and adaptive control," Journal of Information and Computational Science, vol. 10, no. 7, pp. 1959-1977, 2013.
[10] T. Tao and H. Z. Xu, "Fault-tolerant control of high-speed trains with parameter uncertainty," Applied Mechanics and Materials, vol. 325-326, pp. 1099-1105, 2013.

[11] X. Yu and J. Jiang, "Hybrid fault-tolerant flight control system design against partial actuator failures," IEEE Transactions on Control Systems Technology, vol. 20, no. 4, pp. 871-886, 2012.

[12] Y. Shen, L. Liu, and E. H. Dowell, "Adaptive fault-tolerant robust control for a linear system with adaptive fault identification," IET Control Theory \& Applications, vol. 7, no. 2, pp. 246-252, 2013.

[13] J. Zhao, B. Jiang, P. Shi, and Z. He, "Fault tolerant control for damaged aircraft based on sliding mode control scheme," International Journal of Innovative Computing, Information and Control, vol. 10, no. 1, pp. 293-302, 2014.

[14] H. Fan, B. Liu, Y. Shen, and W. Wang, "Adaptive failure compensation control for uncertain systems with stochastic actuator failures," IEEE Transactions on Automatic Control, vol. 59, no. 3, pp. 808-814, 2014.

[15] S. Yin, H. Luo, and S. X. Ding, "Real-time implementation of fault-tolerant control systems with performance optimization," IEEE Transactions on Industrial Electronics, vol. 61, no. 5, pp. 2402-2411, 2014.

[16] X. Y. Zhang, B. Jiang, and K. Zhang, "A reliable tracking control for the 3-DOF hovering system of quadrotor with multiactuator faults," Chinese Conference of Decision and Control, pp. 4961-4966, 2013.

[17] Q. Hu, X. Huo, B. Xiao, and Z. Zhang, "Robust finite-time control for spacecraft attitude stabilization under actuator fault," Proceedings of the Institution of Mechanical Engineers. Part I: Journal of Systems and Control Engineering, vol. 226, no. 3, pp. 416-428, 2012.

[18] W. Chen and F. N. Chowdhury, "Design of sliding mode observers with sensitivity to incipient faults," in Proceedings of the 16th IEEE International Conference on Control Applications (CCA '07), pp. 795-800, Singapore, October 2007.

[19] M. Corless and J. A. Y. Tu, "State and input estimation for a class of uncertain systems," Automatica, vol. 34, no. 6, pp. 757-764, 1998.

[20] M. R. Soltanpour, B. Zolfaghari, M. Soltani, and M. H. Khooban, "Fuzzy sliding mode control design for a class of nonlinear systems with structured and unstructured uncertainties," International Journal of Innovative Computing, Information and Control, vol. 9, no. 7, pp. 2713-2726, 2013.

[21] X. Zhang, T. Parisini, and M. Polycarpou, "Sensor bias fault isolation in a class of nonlinear systems," IEEE Transactions on Automatic Control, vol. 50, no. 3, pp. 370-376, 2005. 


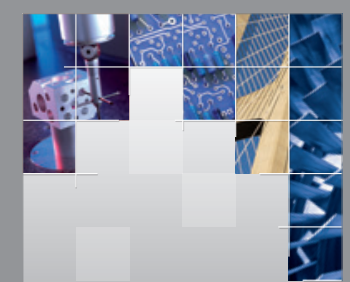

\section{Enfincering}
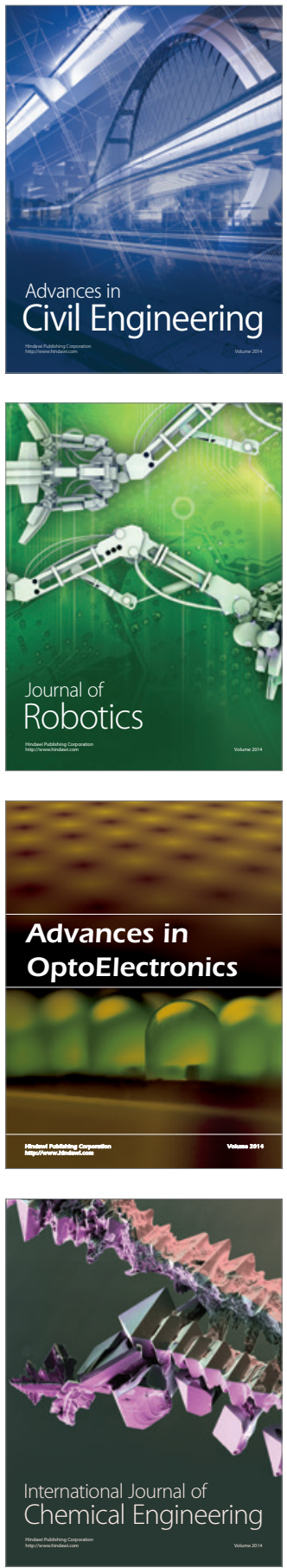

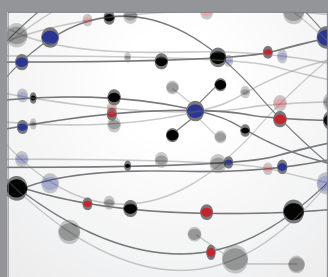

The Scientific World Journal

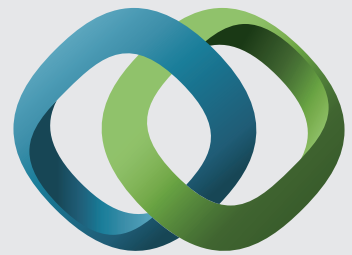

\section{Hindawi}

Submit your manuscripts at

https://www.hindawi.com
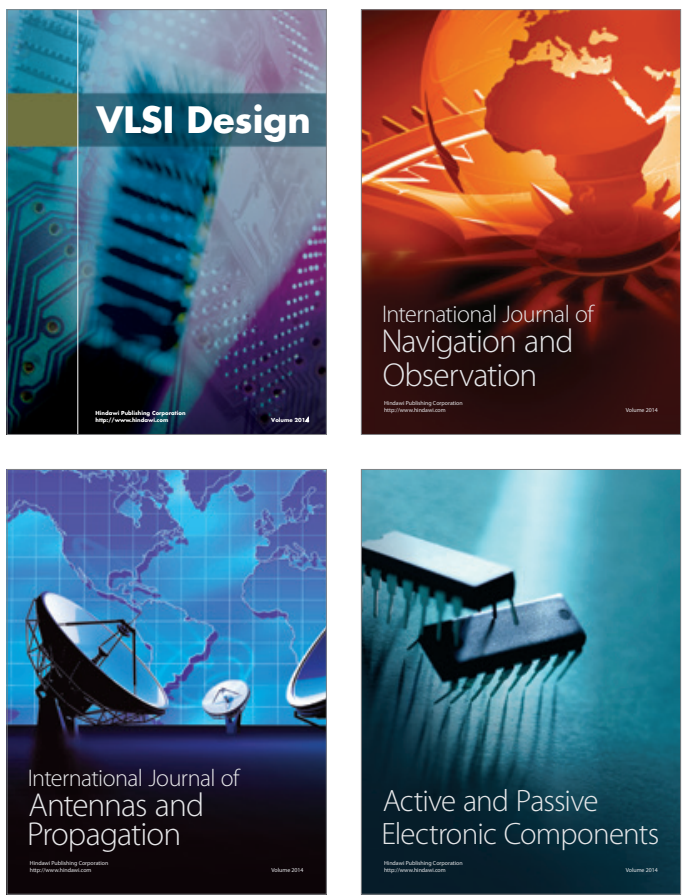
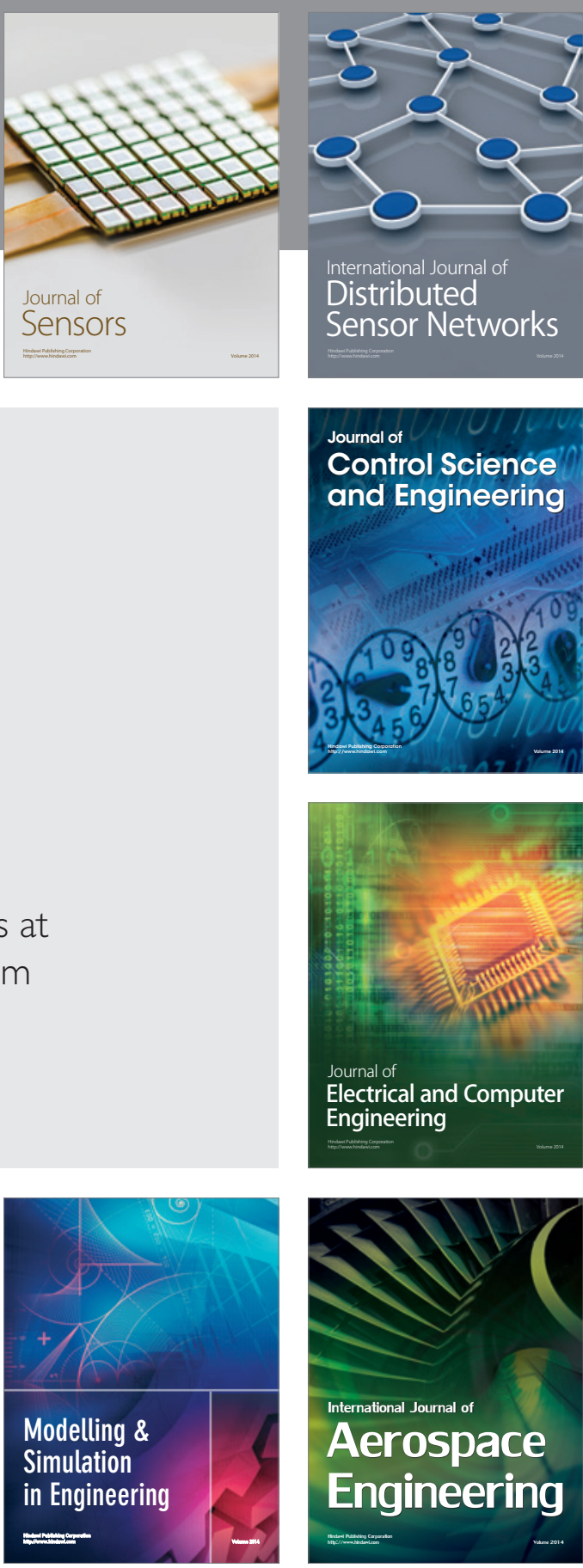

International Journal of

Distributed

Sensor Networks

$-$

Joumal of

Control Science

and Engineering
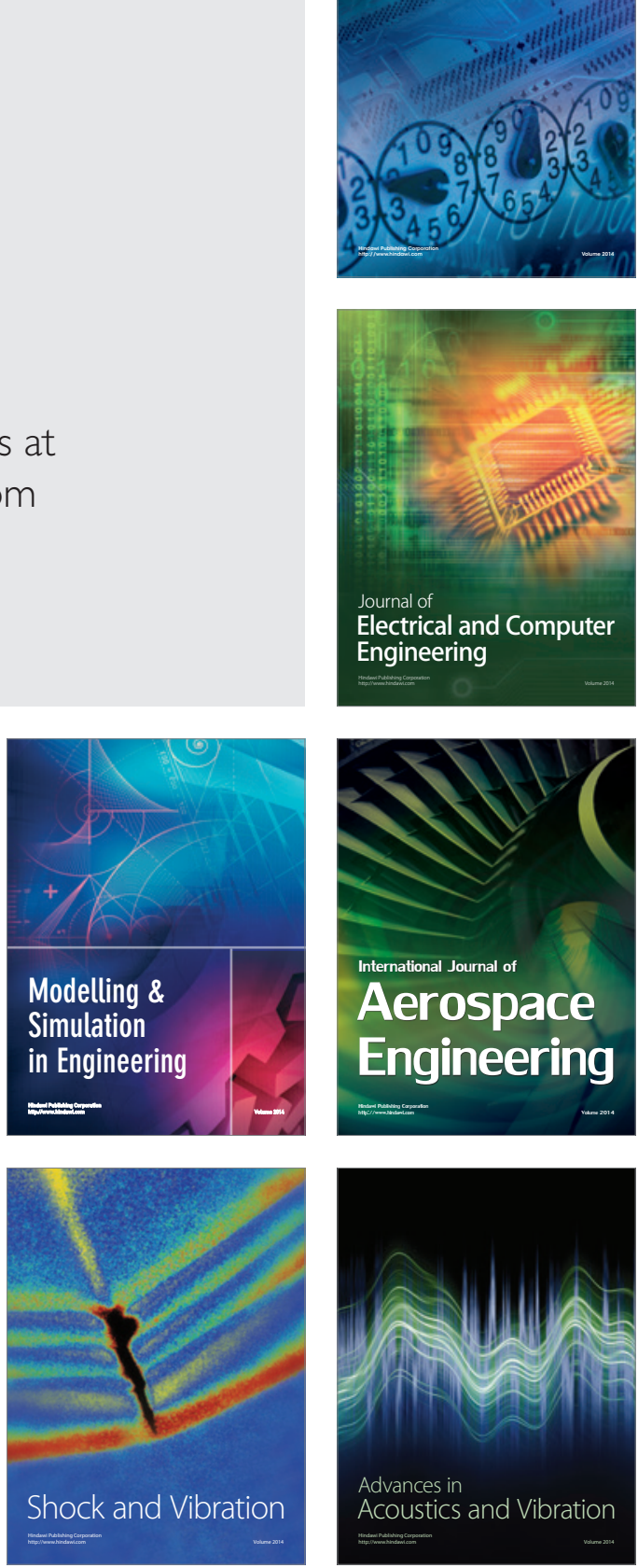